Damit hätte ich mich im wesentlichen meiner, wie ich gestehen muß nicht sehr befriedigenden Aufgabe erledigt. Im Hinblick auf eine zukünftige statistische Bearbeitung und zugleich zur Rechtfertigung meiner abweichenden Stellungnahme gegenüber der Fassung des Fragebogens, an der ich nicht beteiligt bin, hätte ich gern die Frage der Ab. grenzung und Bezeichnung der einzelnen Abschnitte des Infektionsvorganges hier wenigstens kurz berührt. Ich füge mich dem dringenden Wunsche unserer Leitung, da ich einsehe, daß die hierdurch bedingte Diskussion bei der Fülle des sonstigen Materials zu weit führen würde, und behalte mir vor, an anderer Stelle hierauf zurückzukommen.

Ich versage es mir daher auch, auf die Literatur hier einzugehen sowie bestimmte Leitsätze zu formulieren, deren Begründung abermals Zeit erfordern würde.

Ich hoffe, daß ich, soweit es bei der Ungleichheit des Materials möglich war, alles Wesentliche herausgeholt habe, und bedaure, da $\$$ die mühevollen Einzelstatistiken einer Anzahl Bearbeiter aus Mangel an größerem gleichwertigen Material nicht die gebührende Ausnutzung erfahren konnten. Ein Ergebnis wird die diesmalige Sammlung trotz aller Mängel insofern haben, als für ein zukünftiges derartiges Unternehmen wertvolle Erfahrungen gesammelt werden konnten, besonders bezüglich der Ausgestaltung des Fragebogens in formaler und technischer Beziehung.

Möge über einer Sammelforschung in späteren Jahren ein günstigeres Gestirn leuchten!

\title{
6. Herr W. Kolle: Über die chemotherapeutische Aktivierung der Salvarsanpräparate auf Grund von Versuchen bei experimenteller Kaninchensyphilis.
}

M. H. Das Problem der Salvarsantherapie ist - wie ich in diesem Kreise nicht besonders zu sagen brauche - nach vielen Richtungen hin noch im Fluß. Sie arbeiten klinisch an der Vervollkommnung und Sicherung der Salvarsantherapie, an der Vermeidung der Schädlichkeiten, wie ich mit zahlreichen Mitarbeitern im Georg Speyer-Hause die gleichen Gesichtspunkte durch experimentelle Arbeiten zu lösen oder zu fördern bemüht bin. Ich will Ihnen hier eine Anzahl der Versuchsergebnisse der letzten 2 Jahre unterbreiten, und wenn ich das tue, so möchte ich eins vorausschicken. Nicht die vorläufigen Ergebnisse der immer fließenden, immer neue Gesichtspunkte und auf Grund neuer Tatsachen die Probleme experimentell und klinisch verfolgenden Forschung stellen allein das Fördernde und Fortschrittverheißende dar, sondern ebenso die.wissenschaftliche Arbeit und Beobachtung des Verlaufs der Versuche. In diesem Sinne möehte ich. Sie an einem Stück Experimentalarbeit teil- 
nehmen lassen, die Ihnen vielleicht für Thre klinischen Maßnahmen nützlich ist, auch wenn die Übertragung der Ergebnisse der Tierversuche auf die klinische Behandlung des syphilitischen Mensehen nicht die gleichen Rẹsultate zeitigen sollten, was erst von Ihnen zu prüfen ist.

Wir haben seit mehr als 3 Jahren uns mit der Wirkung der Metalle bei Kaninchensyphilis beschäftigt. Teh möchte Thnen zunächst die Wirkung des Quecksilbers allein und in Kombination mit Salvarsa $n$ präparaten mitteilen und die Wirkungsweise gegenüberstellen derjenigen der echten Metallsalvarsane, darunter eines neuen Präparates, des Neosilbersalvarsans. Alle Versuche wurden mit dem schon von Paul Ehrlich und Hata benutzten und im Speyer-Hause weiter fortgezüchteten Truffistamm angestellt.

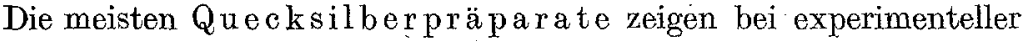
Kaninchensyphilis eine Wirkung auf die Spirochäten nur bei Dosen, die ganz in der Nähe der tödlichen liegen oder mit ihr zusammenfallen.

In Fortsetzung früherer Untersuchungen wurde von mehr als 30 Quecksilberpräparaten zunächst die Toxizität für Kaninchen in großen Versuchsreihen bestimmt. Von den am meisten in der Praxis gebräuchlichen wurde außerdem die Heiliwirkung bei Kaninchensyphilis geprüft. Die Bestimmung der tödlichen Dosis aller Quecksilberpräparate stößt. bei Kaninchen bekanntlich auf große Schwierigkeiten, weil diese Tierart für $\mathrm{Hg}$ sehr empfänglich ist und weil neben einer akuten $\mathrm{Hg}$-Giftwirkung leicht eine chronische Vergiftung infolge besonderer Empfindlichkeit der Kaninchen auch nach kleinen Dosen der $\mathrm{Hg}$-Präparate eintritt. Durch große Versuchsreihen läßt sich indessen die Dosis tolerata sowie die Dosis letalis mit genügender Sicherheit ermitteln ${ }^{1}$ ).

Unter allen untersuchten $\mathrm{Hg}$-Verbindungen sind bis jetzt wenige gefunden worden, die auch in sicher erträglichen Dosen ein Verschwinden der Spirochäten und sogar ein Ausheilen der Schanker herbeiführen, d. h. einen, wenn auch geringen chemotherapeutischen Index aufweisen. Das eine der Präparate (BiHg 52) konnte allerdings bei der chemischen Darstellung nicht konstant in gleicher Zusammensetzung gewonnen werden, während die andere Verbindung ( $\mathrm{Vu}$. VI) vielleicht einen Ausgangspunkt für die Darstellung therapeutiseh wirksamer Quecksilberverbindungen geben wird.

Die Heilung der Primäraffekte bei Kaninchensyphilis vollzieht sich, wenn sie überhaupt erfolgt, nach Anwendung von $\mathrm{Hg}$-Präparaten anders als nach Injektion von Arsenobenzolderivaten. Selbst bei den erwähnten zwei Präparaten, bei denen die Spirochäten vom 3. Tage nach Einverleibung von Dosen, die geringer als die erträgliche waren, bei der Mehrzahl der Tiere dauernd zum Verschwinden gebracht und die Heilung der Schanker herbeigeführt wurden, geht die Ausheilung der Primär-

1) Kolle u. Ritz, Dtsch. med. Wochensehr. 1919. 
affekte viel langsamer als nach Anwendung von Arsenobenzolderivaten von statten. Auch die Resorption der Schanker erfolgt, ohne daß die Spirochäten wieder auftreten, fast so langsam wie bei den spontan sich zurückbildenden Schankern, die man ja gelegentlich im späteren Stadium der experimentellen Syphilisinfektion der Kaninchen - nach 4-6 monatigem Bestehen der Schanker - beobachten kann.

Im Gegensatz zu den Quecksilberpräparaten erfolgt nach Anwendung der Salvarsanpräparate fast stets rasche Resorption des syphilitischen Gewebes ohne Detritusbildung. Nach Salvarsan sind Lokalrezidive selten, nach $\mathrm{Hg}$ Reindurationen häufig beim Kaninchen.

Wir können trotzdem auf Grund solcher Beobachtungen die Hoffnung hegen, daß wir vielleicht doch noch zu praktisch brauchbaren, sicher chemotherapeutisch bei Kaninchensyphilis wirksamen Quecksilberpräparaten gelangen, wenn man das von den genannten Verbindungen auch noch nicht sagen kann. Denn in Analogie mit den Arsenikalien, bei denen die Reduktion des fünfwertigen Arsens zum dreiwertigen die therapeutische Wirksamkeit mit sich bringt, liegt es nahe, anzunehmen, daß auch bei organischen $\mathrm{Hg}$-Verbindungen der Utbergang von der zweiwertigen zur einwertigen Form bzw. die Gewinnung von präformierten oder im Körper entstehenden einwertigen Quecksilberverbindungen, den Mercuroverbindungen, das therapeutische Prinzip wirksamer Hg-Körper darstellt. Aber die bisher bekannten, Ihnen hier kurz in ihrer Wirksamkeit skizzierten Quecksilberverbindungen sind bisher nicht zu vergleichen in ihrer Wirksamkeit mit derjenigen der Arsenobenzole. Allerdings ist das Kaninchen gerade für Quecksilber ja ein relativ wenig geeignetes Tier; um so größere Bedeutung dürften aber etwa beobachtete, auch nur geringe, sicherechemotherapeutische Effekte darstellen. Ich werde über die mit Dr. Ritz begonnenen, mit Dr. Ru p pert unter Mitarbeit der Laboratoriumsassistentin Frl. Möbus fortgesetzten Studien mit Quecksilberpräparaten später eingehend Mitteilung machen.

Mit Rücksicht auf die von vielen Klinikern immer wieder betonte Notwendigkeit, bei der Behandlung der menschlichen Syphilis das $\mathbf{H g}$ zur Unterstützung der Salvarsantherapie nicht entbehren zu können, sowie andererseits infolge der allgemein anerkannten mächtigen Wirkung, die die Metallsalvarsane, namentlich das Silbersalvarsan; auf die Spirochäten und die Manifestationen der menschlichen Syphilis entfalten, ist von mir die Frage der Wirkung von Hg - Präparaten in Kombination mit Salvarsanpräparaten bei experimenteller Kaninchensyphilis erneut mit Dr. Ruppert und der Laboratoriumsassistentin Frl. Möbus in Bearbeitung genommen worden.

Die Ausführung der kombinierten Behandlung syphilitischer Kaninchen mit stark ausgeprägten Primäraffekten geschah nach den gleichen 
W. Kolle: Über die chemotherapeutische Aktivierung der

Dosis tox. 0,04 .

Dosis tol. 0,025

Tabelle I.

\begin{tabular}{|c|c|c|c|c|c|c|}
\hline \multirow{2}{*}{$\begin{array}{l}\text { Kanin- } \\
\text { chen } \\
\text { Nr. }\end{array}$} & \multirow{2}{*}{$\begin{array}{l}\text { Durchmesser der } \\
\text { Schanker } \\
\text { Spirochäten vor } \\
\text { Iniektion }\end{array}$} & \multirow{2}{*}{ Dosis pro Kilo } & \multicolumn{2}{|c|}{ 1. Tag } & \multicolumn{2}{|c|}{ 2. Tag } \\
\hline & & & $\begin{array}{l}\text { Spirochäten- } \\
\text { befund }\end{array}$ & $\begin{array}{l}\text { Schanker- } \\
\text { durchresser }\end{array}$ & $\begin{array}{l}\text { Spirochäten- } \\
\text { befund }\end{array}$ & $\begin{array}{c}\text { Schanker- } \\
\text { durchmesser }\end{array}$ \\
\hline 784 & $\begin{array}{l}\text { R. } 3,1 \times 3,1 \times 2,0 \\
\text { L. } 3,3 \times 3,4 \times 2,0 \\
\text { R. }+ \text { g. bew. } \\
\text { L. + + g. bew. }\end{array}$ & $\begin{array}{l}0,045 \\
(0,0045 \mathrm{Hg})\end{array}$ & $\begin{array}{l}+ \text { g. bew. } \\
+ \text { g. bew. }\end{array}$ & $\begin{array}{l}3,0 \times 3,4 \times 2,0 \\
3,3 \times 3,4 \times 2,0\end{array}$ & $\begin{array}{r}+ \text { w. bew. } \\
++ \text { g. bew. }\end{array}$ & $\begin{array}{l}3,2 \times 3,4 \times 2,1 \\
3,4 \times 3,6 \times 2,1\end{array}$ \\
\hline 804 & $\begin{array}{c}1,5 \times 1,5 \times 1,0 \\
+++\mathrm{g}, \text { bew. }\end{array}$ & $\begin{array}{l}0,035 \\
(0,0035 \mathrm{Hg})\end{array}$ & $+t+$ g. bew. & $1,6 \times 1,6 \times 1,1$ & +++ g. bew. & $1,5 \times 1,6 \times 1,1$ \\
\hline 798 & $\begin{array}{l}\text { R. } 1,1 \times 1,3 \times 0,8 \\
\text { L. } 1,7 \times 2,2 \times 1,5 \\
\text { R. }+ \text { g. bew. } \\
\text { L. }++ \text { g. bew. }\end{array}$ & $\begin{array}{l}0,08 \\
(0,003 \mathrm{Hg})\end{array}$ & $\begin{array}{r}+ \text { w. g. bew. } \\
+ \text { g. bew. }\end{array}$ & $\begin{array}{l}1,1 \times 1,3 \times 0,8 \\
1,8 \times 2,2 \times 1,4\end{array}$ & $\begin{array}{l}+ \text { s. w. bew. } \\
+ \text { w. bew. }\end{array}$ & $\begin{array}{l}1,2 \times 1,3 \times 0,7 \\
1,9 \times 2,2 \times 1,4\end{array}$ \\
\hline 727 & $\begin{array}{c}1,4 \times 1,4 \times 0,7 \\
+++ \text { g. bew. }\end{array}$ & $\begin{array}{l}0,025 \\
(0,0025 \mathrm{Hg})\end{array}$ & + y. bew. & $1,4 \times 1,4 \times 0,9$ & + w. g. bew. & $1,5 \times 1,4 \times 0,9$ \\
\hline 733 & $\begin{array}{l}\text { R. } 1,5 \times 1,9 \times 1,1 \\
\text { L. } 1,3 \times 1,5 \times 1,0 \\
\text { R. }++ \text { g. bew. } \\
\text { L. }+++ \text { g. bew. }\end{array}$ & $\begin{array}{l}0,02 \\
(0,002 \mathrm{Hg})\end{array}$ & $\begin{array}{r}+ \text { g. bew. } \\
++ \text { g. bew. }\end{array}$ & $\begin{array}{l}1,4 \times 1,7 \times 1,2 \\
1,4 \times 1,5 \times 1,0\end{array}$ & $\begin{array}{l}++ \text { g. bew. } \\
+t \text { g. bew. }\end{array}$ & $\begin{array}{l}1,3 \times 1,4 \times 1,2 \\
1,4 \times 1,5 \times 1,0\end{array}$ \\
\hline 758 & $\begin{array}{r}\text { R. } 1,3 \times 1,6 \times 1,0 \\
\text { L. } 1,6 \times 2,0 \times 1,2 \\
\text { R. }++ \text { g. bew. } \\
\text { L. }+++ \text { g. bew. }\end{array}$ & $\begin{array}{l}0,01 \\
(0,001 \mathrm{Hg})\end{array}$ & $\begin{array}{c}+ \text { bew. } \\
+++ \text { g. bew. }\end{array}$ & $\begin{array}{l}1,4 \times 1,6 \times 1,0 \\
1,7 \times 2,0 \times 1,1\end{array}$ & - & 一 \\
\hline
\end{tabular}

Tabelle II.

Dosis tox. 0,003

Dosis tol. $0,008-0,007$

Wirkung von "Hg colloidale" auf des

\begin{tabular}{|c|c|c|c|c|c|c|}
\hline \multirow{2}{*}{$\begin{array}{c}\text { Kanin- } \\
\text { ehen } \\
\text { Nr. }\end{array}$} & \multirow{2}{*}{$\begin{array}{l}\text { Durchmesser der } \\
\text { Schanker } \\
\text { Spirochäten vor } \\
\text { Injektion }\end{array}$} & \multirow{2}{*}{ Dosis pro Kilo } & \multicolumn{2}{|c|}{ 1. Tag } & \multicolumn{2}{|c|}{ 2. Tag } \\
\hline & & & $\begin{array}{l}\text { Spirochäten- } \\
\text { befund }\end{array}$ & $\begin{array}{l}\text { Schanker- } \\
\text { durchmesser }\end{array}$ & $\begin{array}{l}\text { Spirochäten- } \\
\text { befund }\end{array}$ & $\begin{array}{c}\text { Schanker- } \\
\text { durchmesser }\end{array}$ \\
\hline 426 & $\begin{array}{c}1,8 \times 2,9 \times 1,2 \\
+ \text { bew. }\end{array}$ & $\begin{array}{l}0,003 \\
(0,0015 \mathrm{Hg})\end{array}$ & + bew. & $1,7 \times 2,8 \times 1,4$ & + w. g. bew. & $1,7 \times 2, \overrightarrow{7} \times 1,2$ \\
\hline 466 & $\begin{array}{l}\text { R.. } 1,8 \times 2,0 \times 1,1 \\
\text { L. } 0,9 \times 1,0 \times 0,7 \\
\text { R. }+ \text { + g. bew. } \\
\text { L. + g. bew. }\end{array}$ & $\begin{array}{l}0,002 \\
(0,001 \mathrm{Hg})\end{array}$ & $\begin{array}{l}\text { R. }+++ \text { unb. } \\
\text { L. }++ \text { g. b. }\end{array}$ & $\begin{array}{l}1,9 \times 2,3 \times 1,3 \\
0,8 \times 1,2 \times 0,8\end{array}$ & $\begin{aligned}+ & + \text { unb. } \\
& + \text { g. bew. }\end{aligned}$ & $\begin{array}{l}1,8 \times 2,1 \times 1,1 \\
0,8 \times 1,1 \times 0,8\end{array}$ \\
\hline 1198 & $\begin{array}{c}2,0 \times 2,0 \times 1,2 \\
+++ \text { g. bew. }\end{array}$ & $\begin{array}{l}0,002 \\
(0,001 \mathrm{Hg})\end{array}$ & ++ bew. & $1,8 \times 2,0 \times 1,2$ & ++ bew. & $1,8 \times 2,0 \times 1,1$ \\
\hline 357 & $\begin{array}{c}\text { R. } 1,8 \times 1,5 \times 0,7 \\
\text { L. } 1,5 \times 1,7 \times 0,7 \\
\text { R. }++ \text { g. bew. } \\
\text { L. }+++ \text { g. bew. }\end{array}$ & $\begin{array}{l}0,001 \\
(0,0005 \mathrm{Hg})\end{array}$ & $\begin{array}{l}+t+g \text { bew. } \\
+t+\text { g. bew. }\end{array}$ & $\begin{array}{l}1,8 \times 1,4 \times 0,8 \\
1,6 \times 1,7 \times 0,7\end{array}$ & $\begin{array}{l}+++ \text { g. bew. } \\
+++ \text { g. bew. }\end{array}$ & $\begin{array}{l}1,8 \times 1,8 \times 1,0 \\
1,7 \times 1,8 \times 0,9\end{array}$ \\
\hline 1116 & $\begin{array}{r}\text { R. } 1,7 \times 1,7 \times 1,0 \\
\text { L. } 1,8 \times 2,4 \times 1,1 \\
\text { R. }++ \text { g. bew. } \\
\text { L. }+++ \text { g. bew. }\end{array}$ & $\begin{array}{l}0,001 \\
(0,0000 \text { 且g) }\end{array}$ & $\begin{array}{l}++ \text { bew. } \\
++ \text { bew. }\end{array}$ & $\begin{array}{l}1,6 \times 1,7 \times 1,0 \\
1,9 \times 2,3 \times 1,3\end{array}$ & $\begin{array}{c}++ \text { g. bew. } \\
++ \text { bew. }\end{array}$ & $\begin{array}{l}1,4 \times 1,7 \times 1,0 \\
1,9 \times 2,2 \times 1,3\end{array}$ \\
\hline
\end{tabular}


Salvarsanpräparate auf Grund von Versuchen bei experim. Kaninchensyphilis. 101

Tabelle I.

auf den Syphilisschanker am Kaninchenhoden.

\begin{tabular}{|c|c|c|c|c|c|c|}
\hline \multicolumn{2}{|c|}{ 3. Tag } & \multicolumn{2}{|c|}{ 4. $\mathrm{Tag}$} & \multicolumn{2}{|c|}{ 5. Tag } & \\
\hline $\begin{array}{l}\text { Spirochäten- } \\
\text { befund }\end{array}$ & $\begin{array}{l}\text { Schanker } \\
\text { durchmesser }\end{array}$ & $\begin{array}{l}\text { Spirochäten- } \\
\text { befund }\end{array}$ & $\begin{array}{c}\text { Schanker- } \\
\text { durchmesser }\end{array}$ & $\begin{array}{l}\text { Spirochäten- } \\
\text { befund }\end{array}$ & $\begin{array}{l}\text { Schanker- } \\
\text { durchmesser }\end{array}$ & \\
\hline $\begin{array}{l}++ \text { g. bew. } \\
++ \text { g. bew. }\end{array}$ & $\begin{array}{l}3,2 \times 3,5 \times 2,3 \\
3,3 \times 3,7 \times 2,1\end{array}$ & $\begin{array}{l}++ \text { g. bew. } \\
++ \text { g. bew. }\end{array}$ & $\begin{array}{l}3, \mathbf{3} \times \mathbf{3 , 5} \times \mathbf{5} \times 2, \mathbf{3} \\
3,4 \times \mathbf{3 , 5} \times 2,0\end{array}$ & - & - & nicht ausgeheilt \\
\hline++ g. bew. & $1,6 \times 1,6 \times 1,2$ & ++ g. bew. & $1,7 \times 1,7 \times 1,1$ & $t+t$ g. bew. & $1,8 \times 1,8 \times 1,1$ & nicht ausgeheilt \\
\hline $\begin{array}{c}\text { + w. g. bew. } \\
\text { + w. g. bew. } \\
\text { vereitert }\end{array}$ & $\begin{array}{l}1,2 \times 1,3 \times 0,6 \\
2,0 \times 2,3 \times 1,5\end{array}$ & - & - & $\begin{array}{l}+ \text { g. bew. } \\
+ \text { w. bew. }\end{array}$ & $\begin{array}{l}1,3 \times 1,3 \times 0,9 \\
2,0 \times 2,6 \times 1,5\end{array}$ & nicht ausgeheilt \\
\hline $\begin{array}{c}\text { +s.w. bew. } \\
\text { (Eiter) }\end{array}$ & $1,6 \times 1,5 \times 0,9$ & - & - & + s. w. bew. & $1,4 \times 1,4 \times 0,8$ & nicht ausgeheilt \\
\hline - & 一 & $\begin{array}{l}++ \text { g. bew. } \\
++ \text { g. bew. }\end{array}$ & $\begin{array}{l}1,3 \times 1,2 \times 0,9 \\
1,3 \times 1,7 \times 0,9\end{array}$ & $\begin{array}{r}++ \text { g. bew. } \\
+++ \text { g. bew. }\end{array}$ & $\begin{array}{l}1,5 \times 1,6 \times 1,1 \\
1,2 \times 1,7 \times 0,9\end{array}$ & nicht ausgeheilt \\
\hline $\begin{array}{r}++ \text { g. bew. } \\
+++ \text { g. bew. }\end{array}$ & $\begin{array}{l}1,6 \times 1,7 \times 1,1 \\
1,8 \times 2,1 \times 1,1\end{array}$ & $\begin{array}{r}+ \text { g. bew. } \\
++ \text { g. bew. }\end{array}$ & $\begin{array}{l}1,7 \times 1,9 \times 1,1 \\
2,1 \times 2,3 \times 1,2\end{array}$ & $\begin{array}{r}++ \text { g. bew. } \\
+++ \text { g. bew. }\end{array}$ & $\begin{array}{l}1,7 \times 2,0 \times 1,0 \\
2,1 \times 2,3 \times 1,3\end{array}$ & nieht ausgeheilt \\
\hline
\end{tabular}

Tabelle II.

Syphilisschanker am Kaninchenhoden.

\begin{tabular}{|c|c|c|c|c|c|c|}
\hline \multicolumn{2}{|c|}{ 3. Tag } & \multicolumn{2}{|c|}{ 4. Tag } & \multicolumn{2}{|c|}{ 5. Tag } & \\
\hline $\begin{array}{l}\text { Spirochäten- } \\
\text { befund }\end{array}$ & $\begin{array}{l}\text { Schanker- } \\
\text { durchmesser }\end{array}$ & $\begin{array}{l}\text { Spirochäten- } \\
\text { befinnd }\end{array}$ & $\begin{array}{c}\text { Schanker- } \\
\text { durchmesser }\end{array}$ & $\begin{array}{l}\text { Spirochäten- } \\
\text { befund }\end{array}$ & $\begin{array}{c}\text { Schanker- } \\
\text { durchmesser }\end{array}$ & \\
\hline + w. g. bew. & $1,6 \times 2,6 \times 1,1$ & + s. w. bew. & $1,6 \times 2,7 \times 1,0$ & + g. bew. & $1,7 \times 2,7 \times 0,9$ & nicht ausgeheilt \\
\hline $\begin{array}{l}++ \text { unb. } \\
++g . \text { bew. }\end{array}$ & $\begin{array}{l}1,7 \times 2,0 \times 1,1 \\
0,8 \times 1,1 \times 0,8\end{array}$ & $\begin{array}{l}\text { + w. bew. } \\
+ \text { bew. }\end{array}$ & $\begin{array}{l}1,6 \times 1,9 \times 1,1 \\
0,9 \times 1,0 \times 0,8\end{array}$ & $\begin{array}{l}+ \text { w. bew. } \\
+ \text { w. bew. }\end{array}$ & $\begin{array}{l}1,5 \times 1,8 \times 1,0 \\
1,0 \times 1 ; 0 \times 0,7\end{array}$ & $\begin{array}{l}\text { am 3. Tag } 0,002 \mathrm{Hg} \\
\text { colloidale, } \\
\text { nicht ausgeheilt }\end{array}$ \\
\hline++ bew. & $1,7 \times 1,9 \times 1,0$ & $t+$ bew. & $1,6 \times 1,8 \times 1,0$ & $t+$ bew. & $1,6 \times 1,8 \times 1,0$ & $\uparrow$ \\
\hline $\begin{array}{r}++ \text { g. bew. } \\
T++ \text { g. bew. }\end{array}$ & $\begin{array}{l}1,8 \times 1,6 \times 1,0 \\
1, \overline{0} \times 1,9 \times 0,8\end{array}$ & $\begin{array}{l}+++ \text { g. bew. } \\
+++ \text { g. bew. }\end{array}$ & $\begin{array}{l}1,7 \times 1,7 \times 0,9 \\
1,5 \times 1,9 \times 0,7\end{array}$ & $\begin{array}{r}t++ \text { g. bew. } \\
++ \text { g. bew. }\end{array}$ & $\begin{array}{l}1,6 \times 1,8 \times 1,0 \\
1,7 \times 1,7 \times 0,8\end{array}$ & $\begin{array}{l}\text { am } 3 \text {. Tag } 0,001 \mathrm{Hg} \\
\text { colloidale, } \\
\text { nieht ausgeheilt }\end{array}$ \\
\hline $\begin{array}{l}T+\text { bew. } \\
t+\text { bew. }\end{array}$ & $\begin{array}{l}1,5 \times 1,7 \times 1,0 \\
1,8 \times 2,2 \times 1,2\end{array}$ & $\begin{array}{l}++ \text { bew. } \\
++ \text { bew. }\end{array}$ & $\begin{array}{l}1,5 \times 1,7 \times 1,0 \\
1,9 \times 2,2 \times 1,2\end{array}$ & $\begin{array}{l}++ \text { bew. } \\
++ \text { bew. }\end{array}$ & $\begin{array}{l}1,5 \times 1,7 \times 1,0 \\
1,7 \times 2,1 \times 1,3\end{array}$ & nicht ausgeheilt \\
\hline
\end{tabular}


Tabelle III.

Wirkung von ,Hg salicyl" anf den

\begin{tabular}{|c|c|c|c|c|c|c|}
\hline \multirow{2}{*}{$\begin{array}{c}\text { Kanin- } \\
\text { chen } \\
\text { Nr. }\end{array}$} & \multirow{2}{*}{$\begin{array}{l}\text { Durchmessser der } \\
\text { Schanker } \\
\text { Spirochäten vor } \\
\text { Injektion }\end{array}$} & \multirow{2}{*}{ Dosís pro Kílo } & \multicolumn{2}{|c|}{ 1. Tag } & \multicolumn{2}{|c|}{ 2. Tag } \\
\hline & & & $\begin{array}{l}\text { Spirochäten- } \\
\text { befund }\end{array}$ & $\begin{array}{l}\text { Schanker- } \\
\text { durchmesser }\end{array}$ & $\begin{array}{l}\text { Spirochäten- } \\
\text { befund }\end{array}$ & $\begin{array}{l}\text { Schanker- } \\
\text { durchmesser }\end{array}$ \\
\hline 262 & $\begin{array}{l}\text { R. } 1,9 \times 2,5 \times 1,5 \\
\text { L. } 1,8 \times 1,8 \times 1,3 \\
\text { R. }++ \text { g. bew. } \\
\text { L. }++ \text { g. bew. } \\
\text { R. } 2,1 \times 2,4\end{array}$ & $\begin{array}{l}0,01 \\
(0,0059 \mathrm{Hg})\end{array}$ & $\begin{array}{l}+ \text { w. bew. } \\
+ \text { w. unbew. }\end{array}$ & $\begin{array}{l}2,0 \times 2,4 \times 1,4 \\
1,8 \times 1,7 \times 1,2\end{array}$ & $\begin{array}{l}+ \text { s. w. f. unb. } \\
+ \text { s. w. unb. }\end{array}$ & $\begin{array}{l}1,6 \times 2,0 \times 1,2 \\
1,7 \times 1,5 \times 1,0\end{array}$ \\
\hline 341 & $\begin{array}{l}\text { L. } 2,0 \times 1,9 \\
\text { R. } \quad+\text { g. bew. } \\
\text { L. + + g. bew. }\end{array}$ & $\begin{array}{l}0,0075 \\
(0,0044 \mathrm{Hg})\end{array}$ & - & - & $\begin{array}{l}++ \text { bew. unb. } \\
+ \text { g. bew. }\end{array}$ & $\begin{array}{l}2,0 \times 20 \\
2,0 \times 1,5\end{array}$ \\
\hline 261 & $\begin{array}{c}2,0 \times 2,3 \times 1,1 \\
+++g . \text { bew. }\end{array}$ & $\begin{array}{l}0,005 \\
(0,0029 \mathrm{Hg})\end{array}$ & + g. bew. & - & +w. bew. & $1,9 \times 2,1 \times 1,0$ \\
\hline 343 & $\begin{array}{c}\text { R. } 2,5 \times 2,5 \\
\text { L. } 3,0 \times 2,6 \\
\text { R. }+++ \text { g. bew } \\
\text { L. }+++ \text { g. bew }\end{array}$ & $\begin{array}{l}0,0025 \\
(0,0015 \mathrm{Hg})\end{array}$ & - & 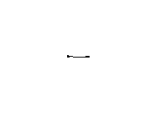 & $\begin{array}{l}++ \text { g. bew. } \\
++ \text { g. bew. }\end{array}$ & $\begin{array}{l}2,7 \times 2,4 \\
2,9 \times 2,3\end{array}$ \\
\hline
\end{tabular}

Tabelle 1V.

Dosis tox. $0,02 \mathrm{ccm}$

Wirkung des „Novasurols“ auf den Syphilis

\begin{tabular}{|c|c|c|c|c|c|c|}
\hline \multirow{2}{*}{$\begin{array}{l}\text { Kanin- } \\
\text { chen } \\
\text { Nr. }\end{array}$} & \multirow{2}{*}{$\begin{array}{l}\text { Durchmesser der } \\
\text { Schanker } \\
\text { Spirochätenbefund } \\
\text { vor Inj"ktion }\end{array}$} & \multirow{2}{*}{ Dosis pro Kito } & \multicolumn{2}{|c|}{ 1. Tag } & \multicolumn{2}{|c|}{ 2. Tag } \\
\hline & & & $\begin{array}{l}\text { Spirochäten- } \\
\text { befund }\end{array}$ & $\begin{array}{l}\text { Schanker- } \\
\text { durchmesser }\end{array}$ & $\begin{array}{l}\text { Spirochäten- } \\
\text { befand }\end{array}$ & $\begin{array}{c}\text { Schanker- } \\
\text { durchmesser }\end{array}$ \\
\hline 672 & $\begin{array}{l}\text { R. } 1,4 \times 1,4 \times 1,1 \\
\text { L. } 1,3 \times 1,4 \times 1,1 \\
\text { R. }++ \text { g. bew. } \\
\text { L. }++ \text { g. bew. }\end{array}$ & 0,005 & $\begin{array}{l}\text { R. + g. bew. } \\
\text { L. + bew. }\end{array}$ & $\begin{array}{l}1,4 \times 1,4 \times 1,7 \\
1,3 \times 1,5 \times 1,1\end{array}$ & $\begin{array}{l}+ \text { g. bew. } \\
+ \text { w. bew. }\end{array}$ & $\begin{array}{l}1,3 \times 1,3 \times 1,1 \\
1,3 \times 1,5 \times 1,1\end{array}$ \\
\hline 661 & $\begin{array}{c}\text { R. } 2,2 \times 2,1 \times 1,2 \\
\text { R. }+++ \text { g. bew. }\end{array}$ & 0,005 & $t+t$ g. bew. & $2,2 \times 2,3 \times 1,2$ & $t++$ g. bew. & $2,3 \times 2,5 \times 1,3$ \\
\hline 685 & $\begin{array}{c}\text { R. } 1,6 \times 1,6 \times 1,1 \\
\text { L. } 1,9 \times 1,8 \times 1,2 \\
\text { R. }+++ \text { g. bew. } \\
\text { L. }++ \text { g. bew. }\end{array}$ & 0,01 & $\begin{array}{l}\text { R. + s. w. bew. } \\
\text { L. + w. bew. }\end{array}$ & $\begin{array}{l}1,7 \times 1,6 \times 1,1 \\
1,8 \times 1,8 \times 1,1\end{array}$ & $\begin{array}{l}+ \text { w. bew. } \\
+ \text { bew. }\end{array}$ & $\begin{array}{l}1,7 \times 1,5 \times 1,0 \\
1,6 \times 1,7 \times 1,0\end{array}$ \\
\hline 759 & $\begin{array}{l}\text { R. } 1,8 \times 1,8 \times 1,0 \\
\text { L. } 1,5 \times 1,5 \times 1,0 \\
\text { R. }+ \text { g. bew. } \\
\text { L. }+ \text { g. bew. }\end{array}$ & 0,015 & $\begin{array}{l}\text { R. + g. bew. } \\
\text { L. + g. bew. }\end{array}$ & $\begin{array}{l}1,7 \times 1,8 \times 1,0 \\
1,5 \times 1,5 \times 1,0\end{array}$ & $\begin{array}{l}+ \text { w. bew. } \\
+ \text { s. w. bew. }\end{array}$ & $\begin{array}{l}1,5 \times 1,7 \times 1,0 \\
1,4 \times 1,5 \times 0,9\end{array}$ \\
\hline 668 & $\begin{array}{l}\text { R. } 1,0 \times 1,1 \times 0,7 \\
\text { L. } 1,8 \times 1,7 \times 1,2 \\
\text { R. }+ \text { g. bew. } \\
\text { L. }++ \text { g. bew. }\end{array}$ & 0,015 & $\begin{array}{l}\text { R. + w. bew. } \\
\text { L. + bew. }\end{array}$ & $\begin{array}{l}1,1 \times 1,1 \times 0,8 \\
1,9 \times 1,8 \times 1,2\end{array}$ & - & - \\
\hline 803 & $\begin{array}{r}\text { R. } 1,5 \times 1,9 \times 0,8 \\
\text { L. } 1,3 \times 1,2 \times 1,0 \\
\text { R. }+ \text { g. bew. } \\
\text { L. }+++ \text { g. bew. }\end{array}$ & 0,0175 & $\begin{array}{l}\text { R. + w. bew. } \\
\text { L. + + g. bew. }\end{array}$ & $\begin{array}{l}1,5 \times 1,9 \times 0,9 \\
1,3 \times 1,3 \times 1,0\end{array}$ & $\begin{array}{c}0 \\
+ \text { s. w. unbew. }\end{array}$ & $\begin{array}{l}1,4 \times 1,7 \times 0,8 \\
1,2 \times 1,2 \times 0,9\end{array}$ \\
\hline 1067 & $\begin{array}{c}\text { R. } 1,5 \times 1,7 \times 1,7 \\
\text { L. } 1,8 \times 1,7 \times 1,0 \\
\text { R. }+++ \text { g bew. } \\
\text { L. +t g. bew. }\end{array}$ & 0,02 & $\begin{array}{l}\text { R. + s. w. unbew. } \\
\text { L. + s.w. unbew. }\end{array}$ & $\begin{array}{l}1,4 \times 1,6 \times 1,4 \\
1,7 \times 1,7 \times 1,1\end{array}$ & - & - \\
\hline
\end{tabular}


Salvarstnpräparate auf Grund von Versuchen bei experim. Kaninchensyphilis. 103

Tabelle III.

Syphilisschanker am Kaninchenhoder.

\begin{tabular}{|c|c|c|c|c|c|c|}
\hline \multicolumn{2}{|c|}{ 3. Tag } & \multicolumn{2}{|c|}{ 4. Tag } & \multicolumn{2}{|c|}{ 5. Tag } & \\
\hline $\begin{array}{l}\text { Spirochäten- } \\
\text { befund }\end{array}$ & $\begin{array}{l}\text { Sehanker- } \\
\text { durchmesser }\end{array}$ & $\begin{array}{l}\text { Spirochäten- } \\
\text { befund }\end{array}$ & $\begin{array}{l}\text { Schanker- } \\
\text { durchmesser }\end{array}$ & $\begin{array}{l}\text { Spirochäten- } \\
\text { befund }\end{array}$ & $\begin{array}{c}\text { Sehanker- } \\
\text { durchmesser }\end{array}$ & \\
\hline $\begin{array}{c}1 \mathrm{unb} . \\
0\end{array}$ & $\begin{array}{l}1,4 \times 1,7 \times 1,1 \\
1,6 \times 1,4 \times 0,9\end{array}$ & 0 & $\begin{array}{l}1,3 \times 1,6 \times 1,0 \\
1,6 \times 1,3 \times 0,8\end{array}$ & 0 & $\begin{array}{l}1,3 \times 1,6 \times 0,9 \\
1,5 \times 1,4 \times 0,7\end{array}$ & $\dagger$ \\
\hline $\begin{array}{l}+ \text { viele unb. } \\
++g \text { bew. }\end{array}$ & $\begin{array}{l}2,0 \times 1,9 \times 1,0 \\
1,9 \times 1,5 \times 0,8\end{array}$ & $\begin{array}{l}\text { + viel unb. } \\
\text { + bew. }\end{array}$ & $\begin{array}{l}1,9 \times 1,9 \times 1,1 \\
1,9 \times 1,6 \times 0,9\end{array}$ & $\begin{aligned}+ & + \text { bew. } \\
& + \text { bew. }\end{aligned}$ & $\begin{array}{l}1,7 \times 1,8 \times 1,1 \\
2,0 \times 1,5 \times 0,9\end{array}$ & nicht ausgeheilt \\
\hline + s. w. umb. & $1,8 \times 1,9 \times 0,9$ & 0 & $1,7 \times 1,9 \times 0,9$ & 0 & $1,6 \times 1,8 \times 0,9$ & am 32. Tag Sp. + \\
\hline $\begin{array}{l}++ \text { g. bew. } \\
++ \text { g. bew. }\end{array}$ & $\begin{array}{l}2,0 \times 2,6 \times 1,0 \\
2,8 \times 2,4 \times 1,0\end{array}$ & $\begin{array}{l}++ \text { g. bew. } \\
++ \text { g. bew. }\end{array}$ & $\begin{array}{l}2,1 \times 2,8 \times 1,2 \\
2.9 \times 2.2 \times 1,1\end{array}$ & $\begin{array}{l}++g \text {. bew. } \\
++ \text { g. bew. }\end{array}$ & $\begin{array}{l}2,3 \times 3,0 \times 1,2 \\
3,1 \times 2,1 \times 1,2\end{array}$ & nicht ausgeheilt \\
\hline
\end{tabular}

Tabelle IV.

schanker am Hoden des Kaninchens.

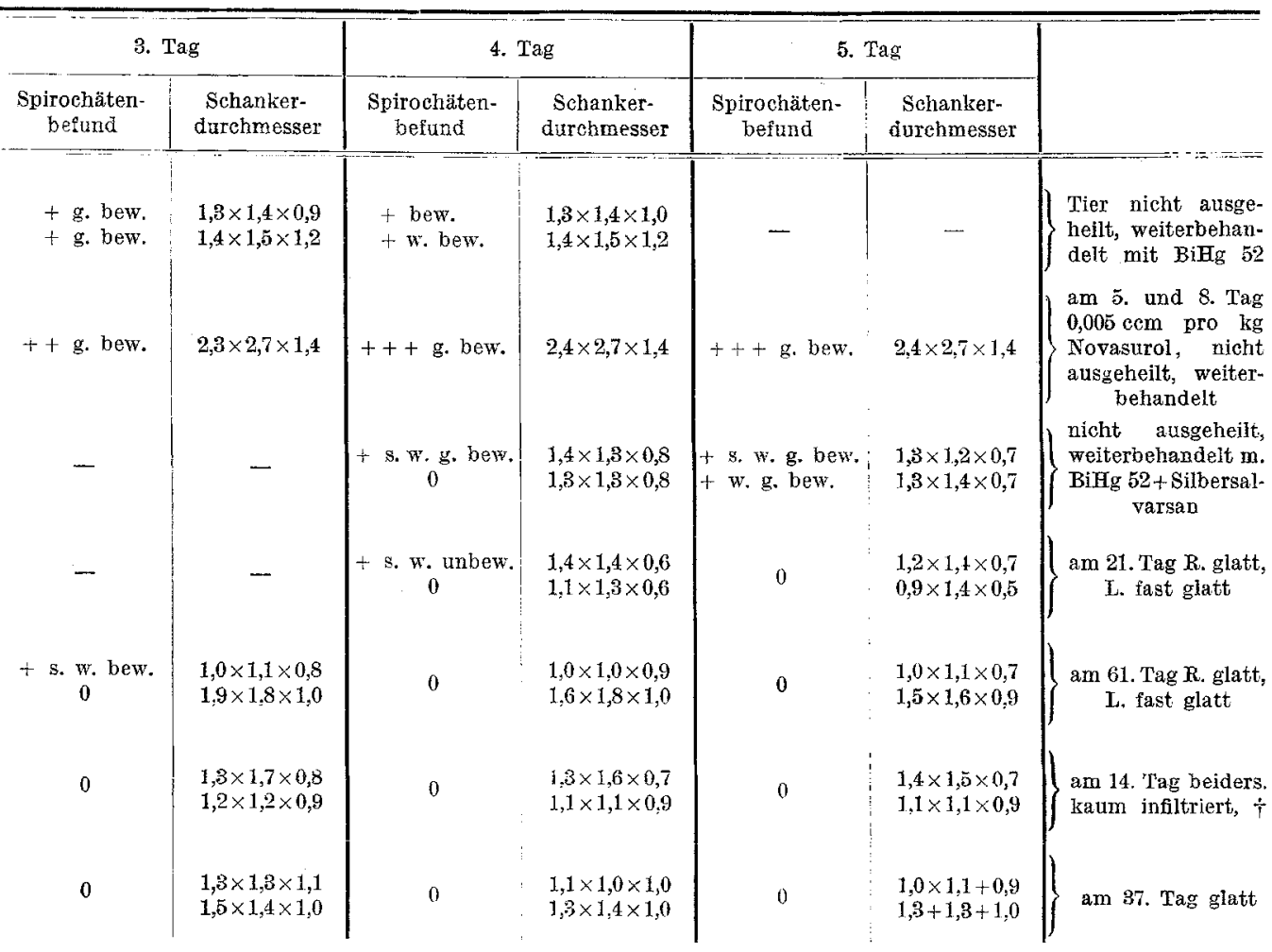




\begin{tabular}{|c|c|c|c|c|c|c|}
\hline \multirow{2}{*}{$\begin{array}{l}\text { Kanin- } \\
\text { chen } \\
\text { Nr. }\end{array}$} & \multirow{2}{*}{$\begin{array}{l}\text { Durchmesser der } \\
\text { Schanker } \\
\text { Spirochäten vor } \\
\text { Injektion }\end{array}$} & \multirow{2}{*}{ Dosis pro Kílo } & \multicolumn{2}{|c|}{ 1. Tag } & \multicolumn{2}{|c|}{ 2. Tag } \\
\hline & & & $\begin{array}{l}\text { Spirochäten- } \\
\text { befund }\end{array}$ & $\begin{array}{l}\text { Schanker- } \\
\text { durchmesser }\end{array}$ & $\begin{array}{l}\text { Spirochäten- } \\
\text { befund }\end{array}$ & $\begin{array}{c}\text { Schanker- } \\
\text { durchmesser }\end{array}$ \\
\hline 1131 & $\begin{array}{l}\text { R. } 2,5 \times 2,7 \times 1,2 \\
\text { L. } 1,8 \times 1,8 \times 0,9 \\
\text { R. }++ \text { g. bew. } \\
\text { L. }+++ \text { g. bew. }\end{array}$ & 0,01 & $\begin{array}{l}+ \text { bew. } \\
++ \text { bew. }\end{array}$ & $\begin{array}{l}2,4 \times 2,7 \times 1,2 \\
1,7 \times 1,7 \times 0,9\end{array}$ & $\begin{array}{c}+ \text { s. w. unb. } \\
0\end{array}$ & $\begin{array}{l}2,0 \times 2,5 \times 1,0 \\
1,5 \times 1,6 \times 0,9\end{array}$ \\
\hline 1132 & $\begin{array}{c}\text { R. } 2,0 \times 2,1 \times 1,3 \\
\text { L. } 2,0 \times 2,2 \times 1,1 \\
\text { R. }+++ \text { g. bew. } \\
\text { L. }+++ \text { g. bew. }\end{array}$ & 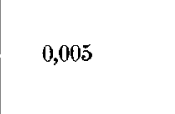 & $\begin{array}{l}+ \text { bew. } \\
++ \text { bew. }\end{array}$ & $\begin{array}{l}2,0 \times 2,1 \times 1,2 \\
1,9 \times 2,2 \times 1,2\end{array}$ & $\begin{array}{l}+ \text { w. bew. } \\
+ \text { s. w. unb. }\end{array}$ & $\begin{array}{l}1,9 \times 1,8 \times 1,1 \\
1,8 \times 1,9 \times 1,2\end{array}$ \\
\hline 1183 & $\begin{array}{c}1,7 \times 2,3 \times 1,1 \\
+++ \text { g. bew. }\end{array}$ & 0,0025 & $+t$ bew. & $1,8 \times 2,3 \times 1,1$ & + w. unbew. & $1,8 \times 2,2 \times 1,1$ \\
\hline
\end{tabular}

\begin{tabular}{|c|c|c|c|c|c|c|}
\hline \multirow{2}{*}{$\begin{array}{l}\text { Kanin- } \\
\text { chen } \\
\text { Nr. }\end{array}$} & \multirow{2}{*}{$\begin{array}{l}\text { Durchmesser der } \\
\text { Schankex } \\
\text { Spirochäten vor } \\
\text { Injektion }\end{array}$} & \multirow{2}{*}{ Dosis pro Kilo } & \multicolumn{2}{|c|}{ 1. Tag } & \multicolumn{2}{|c|}{ 2. Tag } \\
\hline & & & $\begin{array}{l}\text { Spirochäten- } \\
\text { befund }\end{array}$ & $\begin{array}{c}\text { Schanker- } \\
\text { durchmesser }\end{array}$ & $\begin{array}{l}\text { Spirochäten. } \\
\text { befund }\end{array}$ & $\begin{array}{l}\text { Schanker- } \\
\text { durchmesser }\end{array}$ \\
\hline 996 & $\begin{array}{l}\text { R. } 1,0 \times 1,3 \times 0,8 \\
\text { L. } 1,5 \times 1,7 \times 1,1 \\
\text { R. }++ \text { g. bew. } \\
\text { L. }++ \text { g. bew. }\end{array}$ & 0,005 & $\begin{array}{l}+ \text { bew. } \\
+ \text { bew. }\end{array}$ & $\begin{array}{l}\mathbf{1}, 0 \times 1,2 \times 0,7 \\
\mathbf{1 , 5} \times 1,7 \times 1,0\end{array}$ & $\begin{array}{l}0 \\
0\end{array}$ & $\begin{array}{l}1,0 \times 1,2 \times 0,7 \\
1,4 \times 1,6 \times 1,0\end{array}$ \\
\hline 830 & $\begin{array}{l}\text { R. } 2,5 \times 3,5 \times 1,7 \\
\text { L. } 2,3 \times 2,5 \times 1,4 \\
\text { R. + g. bew. } \\
\text { L. }+++ \text { g. bew. }\end{array}$ & 0,004 & $\begin{array}{l}+ \text { w. bew. } \\
+ \text { bew. }\end{array}$ & $\begin{array}{l}2,5 \times 3,5 \times 1,7 \\
2,4 \times 2,5 \times 1,4\end{array}$ & $\begin{array}{l}+ \text { s. w. unb. } \\
+ \text { s. w. bew. }\end{array}$ & $\begin{array}{l}2,5 \times 3,5 \times 1,6 \\
2,3 \times 2,6 \times 1,4\end{array}$ \\
\hline 1141 & $\begin{array}{l}\text { R. } 1,9 \times 1,9 \times 1,2 \\
\text { L. } 1,7 \times 1,8 \times 1,1 \\
\text { R. }++ \text { g. bew. } \\
\text { L. }++ \text { g. bew. }\end{array}$ & 0,003 & $\begin{array}{l}+ \text { bew. } \\
+ \text { bew. }\end{array}$ & $\begin{array}{l}2,0 \times 1,9 \times 1,3 \\
1,6 \times 1,7 \times 1,1\end{array}$ & $\begin{array}{l}+ \text { s. w. unb. } \\
+ \text { w. unb. }\end{array}$ & $\begin{array}{l}1,8 \times 1,8 \times 1,2 \\
1,5 \times 1,7 \times 1,0\end{array}$ \\
\hline 1189 & $\begin{array}{l}1,5 \times 1,8 \times 1,3 \\
+++ \text { g. bew. }\end{array}$ & 0,002 & ++ bew. & $1,6 \times 1,8 \times 1,4$ & + bew. & $1,5 \times 1,9 \times 1,3$ \\
\hline 1119 & $\begin{array}{c}1,8 \times 2,3 \times 1,3 \\
++ \text { g. bew. }\end{array}$ & 0,001 & $t+$ bew. & $2,0 \times 2,3 \times 1,5$ & + bew. & $2,0 \times 2,3 \times 1,3$ \\
\hline
\end{tabular}

Gesichtspunkten, wie die Bestimmung der Wertigkeit der Salvarsanpräparate und zwar wurde nach Analogie der Versuche von Linser die intravenöse Injektion von Gemischen verschiedener Salvarsanpräparate mit Quecksilberverbindungen herangezogen. Von den Hg-Präparaten wurde zunächst das von Linser für Salvarsan-Hg-Gemische empfohlene Sublimat herangezogen, später gingen wir zur Kombination mit anderen löslichen Hg-Verbindungen über und haben das auch klinisch schon erprobte Novasurol als sehr geeignet für die Mischung mit Salvarsanen gefunden.

Sowohl das Sublimat wie das Novasurol wirken, wie aus den Tabellen ersichtlich, erst in höheren Dosen auf die Spirochäten der Kaninchen- 
Salvarsanpräparate auf Grund von Versuchen bei experim. Kaninchensyphilis. 105

Tabelle V.

Syphilisschanker am Hoden des Kaninchens.

\begin{tabular}{|c|c|c|c|c|c|c|}
\hline \multicolumn{2}{|c|}{ 3. Tag } & \multicolumn{2}{|c|}{ 4. Tag } & \multicolumn{2}{|c|}{ 5. Tag } & \\
\hline $\begin{array}{l}\text { Spirochäten- } \\
\text { befund }\end{array}$ & $\begin{array}{l}\text { Schanker- } \\
\text { durchmesser }\end{array}$ & $\begin{array}{l}\text { Spirochäten- } \\
\text { befund }\end{array}$ & $\begin{array}{c}\text { Schanker- } \\
\text { durchmesser }\end{array}$ & $\begin{array}{l}\text { Spirochäten- } \\
\text { befund }\end{array}$ & $\begin{array}{l}\text { Schanker- } \\
\text { durchmesser }\end{array}$ & \\
\hline $\begin{array}{l}0 \\
0\end{array}$ & $\begin{array}{l}2,0 \times 2,2 \times 1,0 \\
1,5 \times 1,5 \times 0,7\end{array}$ & $\begin{array}{l}0 \\
0\end{array}$ & $\begin{array}{l}1,7 \times 2,0 \times 1,0 \\
1,3 \times 1,4 \times 0,6\end{array}$ & $\begin{array}{l}0 \\
0\end{array}$ & $\begin{array}{l}1,8 \times 2,0 \times 1,0 \\
1,5 \times 1,1 \times 0,6\end{array}$ & am 5ิ. Tage glatt \\
\hline $\begin{array}{l}0 \\
0\end{array}$ & $\begin{array}{l}1,6 \times 1,7 \times 1,0 \\
1,6 \times 1,7 \times 1,0\end{array}$ & $\begin{array}{l}0 \\
0\end{array}$ & $\begin{array}{l}1,7 \times 1,7 \times 1,0 \\
1, \tilde{0} \times 1,7 \times 1,0\end{array}$ & $\begin{array}{l}0 \\
0\end{array}$ & $\begin{array}{l}1,5 \times 1,5 \times 1,0 \\
1,3 \times 1,3 \times 1,0\end{array}$ & am 22. Tage $\mathrm{Sp}$ \\
\hline 0 & $1,8 \times 2,0 \times 1,0$ & 0 & $1,5 \times 1,7 \times 1,1$ & 0 & $1,3 \times 1,6 \times 1,0$ & am 44. Tage Sp. \\
\hline
\end{tabular}

Tabelle VI.

Syphilisschanker am Hoden des Kaninchens.

\begin{tabular}{|c|c|c|c|c|c|c|}
\hline \multicolumn{2}{|c|}{ 3. Tag } & \multicolumn{2}{|c|}{ 4. Tag } & \multicolumn{2}{|c|}{ 5. Tag } & \\
\hline $\begin{array}{l}\text { Spirochäten- } \\
\text { befund }\end{array}$ & $\begin{array}{l}\text { Schanker- } \\
\text { durchmesser }\end{array}$ & $\begin{array}{l}\text { Spirochäten- } \\
\text { befund }\end{array}$ & $\begin{array}{l}\text { Schanker. } \\
\text { durchmesser }\end{array}$ & $\begin{array}{l}\text { Spirochäten- } \\
\text { befund }\end{array}$ & $\begin{array}{c}\text { Schanker- } \\
\text { durchmesser }\end{array}$ & \\
\hline $\begin{array}{l}0 \\
0\end{array}$ & $\begin{array}{l}1,0 \times 1,1 \times 0,7 \\
1,3 \times 1,5 \times 1,0\end{array}$ & $\begin{array}{l}0 \\
0\end{array}$ & $\begin{array}{l}0,9 \times 1,0 \times 0,7 \\
1,8 \times 1,4 \times 1,0\end{array}$ & $\begin{array}{l}0 \\
0\end{array}$ & $\begin{array}{l}1,0 \times 1,0 \times 0,7 \\
1,1 \times 1,3 \times 1,0\end{array}$ & am 22. Tage Sp. $\dagger$ \\
\hline $\begin{array}{c}0 \\
+ \text { w. bew. }\end{array}$ & $\begin{array}{l}2,3 \times 3,8 \times 1,5 \\
2,8 \times 2,5 \times 1,2\end{array}$ & $\begin{array}{c}0 \\
+\quad \text { w. bew. }\end{array}$ & $\begin{array}{l}2,2 \times 3,3 \times 1,5 \\
2,3 \times 2,4 \times 1,3\end{array}$ & $\begin{array}{l}0 \\
0\end{array}$ & $\begin{array}{l}2,3 \times 3,8 \times 1,5 \\
2,8 \times 2,4 \times 1,4\end{array}$ & am 64. Tage glatt \\
\hline $\begin{array}{l}0 \\
0\end{array}$ & $\begin{array}{l}1,5 \times 1,7 \times 1,1 \\
1,5 \times 1,6 \times 1,0\end{array}$ & $\begin{array}{l}0 \\
0\end{array}$ & $\begin{array}{l}1,6 \times 1,6 \times 1,1 \\
1,3 \times 1,4 \times 0,9\end{array}$ & $\begin{array}{l}0 \\
0\end{array}$ & $\begin{array}{l}1,5 \times 1,4 \times 1,0 \\
1,3 \times 1,4 \times 1,0\end{array}$ & am 80. Tage glatt \\
\hline t w. bew. & $1,5 \times 1,8 \times 1,2$ & + bew. & $1,5 \times 2,0 \times 1,2$ & + bew. & $1,5 \times 2,0 \times 1,1$ & $\dagger$ \\
\hline + s. w. unb. & $1,9 \times 2,1 \times 1,2$ & 0 & $1,7 \times 2,0 \times 1,2$ & + w. g. bew. & $1.7 \times 2,0 \times 1,2$ & nicht ausgeheilt \\
\hline
\end{tabular}

syphilis und führen zu einer Verkleinerung der Schanker, in ganz seltenen Fällen auch zu einer Ausheilung. Diese Dosen sind aber so hoch, daß sie mit der erträglichen bzw. tödlichen Dosis zusammenfallen.

Es wurde hierbei die beachtenswerte und unerwartete Feststellung gemacht, daß syphilitische Kaninchen alle $\mathrm{Hg}$ - Präparate besser vertragen als normale Tiere. Wir haben hier eine Analogie zur Toleranz der Paralytiker gegen Salvarsan, während umgekehrt Sekundärsyphilitiker entschieden empfindlicher als normale Menschen gegen die Arsenobenzolderivate sind.

Bei den Mischungen des Sublimats sowohl wie des Novasurols mit den verschiedenen Salvarsanpräparaten kann es sich nicht um Analoga der 
Metallsalvarsane handeln. Denn die Mischungen von Salvarsan und $\mathrm{Hg}$ bleiben nur ganz kurze Zeit klar, selbst wenn man kleinste Mengen $\mathrm{Hg}$ dem Salvarsan zusetzt. Bei Verwendungen größerer Mengen von $\mathrm{Hg}$ erfolgt Trübung der Gemische (,Tyndallphänomen“) und daran anschlieBend Ausfällung.

Schon dadurch unterscheiden sich die Quecksilbersalvarsanmischungen von den löslichen Metallsalvarsanverbindungen, z. B. dem Kupferund Silbersalvarsan, aus denen Abscheidung von metallischem oder colloidalem Kupfer bzw. Silber nur nach eingreifenden chemischen Zersetzungsmaßnahmen erfolgt. Bei den Metallsalvarsanen: Kupfer-, Gold-, Silbersalvarsan sind, wie aus den Untersuchungen von Ehrlich und $\mathrm{Karrer}{ }^{1}$ ), Binz, Bauer und Hallstein ${ }^{2}$ ) hervorgeht, die Metalle mit dem Salvarsanmolekül in chemischer Bindung. Wir haben in den Metallsalvarsanen neueeinheitliche, lösliche chemische Körper mit neuen chemotherapeutischen Eigenschaften vor uns. Um die Bildung solcher löslicher Verbindungen kann es sich aber bei der Mischung von Salvarsanpräparaten mit Hg-Verbindungen nicht handeln.

Die chemischen Reaktionen, die sich bei der Mischung von $\mathrm{HgCl}_{2}$ - und es gilt das gleiche für fast alle bekannten $\mathrm{Hg}$-Verbindungen mit Arsenoverbindungen abspielen, sind verschieden, je nachdem man die $\mathrm{Hg}$-Verbindung, z. B. das $\mathrm{HgCl}_{2}$, oder das Salvarsan in Überschuß in den Mischungen hat. Auch sind die Reaktionen verschieden, je nachdem Altsalvarsan bzw. Silbersalvarsan oder Methylensulfoxylatarsenobenzolderivate, z. B. Neosalvarsan oder Sulfoxylatsalvarsan benutzt werden. Setzt man - wie aus Mitteilung des Herrn Dr. Kircher and Dipl.-Ing. v. Ruppert über die in den Laboratorien der Farbwerke vorm. Meister Lucius \& Brüning, Höchst a. M. ${ }^{3}$ ) ausgeführten Untersuchungen hervorgeht - Sublimat stark im ÜberschuB zu Altsalvarsan (Verhältnis $4 \mathrm{HgCl}_{2}$ zu 1 Salv.), so tritt eine Reaktion ein, bei der Calomel, Chlorwasserstoff und freie arsenige Säure entstehen. Bei der sog. Linserschen Totalfällung $\left(\mathrm{HgCl}_{2}\right.$ und Neosalvarsan) ist, wenn ein großer Überschuß von $\mathrm{HgCl}_{2}$ genommen wird, das gleiche der Fall. Die unlösliche Verbindung, die sich in diesen Fällen abscheidet, ist nicht, wie Bülow annahm, eine unlösliche organische Quecksilberverbindung, sondern Calomel nebst kleinen Mengen metallischen Quecksilbers, während das Arsen als arsenige Säure abgespalten wird (Kircher und v. Ruppert).

Wie die Untersuchungen von Binz und $\mathrm{Bauer}{ }^{4}$ ) ergeben haben,

1) Ber. d. Deutsch. chem. Ges. 48, 1634. 1915.

2) Ber. d. Deutsch. chem. Ges. 53, 416. 1920.

3) Privatmitteilang.

4) Zeitschr. f. angew. Chemie 34, 223. 1921. 
handelt es sich bei der Mischung von Sublimat mit Salvarsan oder Neosalvarsan um eine größere Anzahl von gleichzeitig stattfindenden Reaktionen. Altsalvarsan wird zu 4-Oxy-3-Aminoarsinoxyd oxydiert, während das Sublimat zu Calomel, oder bei Anwendung kleiner Sublimatmengen, zu metallischem Quecksilber reduziert wird. Bei Anwendung von Neosalvarsan wird dem Sublimat außer der Arsenogruppe auch noch die ebenfalls stark reduzierend wirkende Sulfoxylatgruppe geboten. Wird dem Neosalvarsan wenig Sublimat, wie es Lins er für seine therapeutischen Injektionen verwendet, zugesetzt, so kann nach Binz und Bauer neben viel unverändertem Neosalvarsan sich noch bilden: kolloides Quecksilber, Arsinoxyde, zum Teil gebunden an Methylensulfoxylsäure oder methylenschwefelige Säure, arsenobenzol-methylenschweflige Säure, Neosalvarsansäure und daneben die aus dem dem Neosalvarsan beigemengten Hyraldit entstandenen formaldehydschweflige Säure und Formaldehydsulfoxylsäure. Die Neosalvarsansäure und die arsenobenzolmethylenschweflige Säure, die unlöslich sind, fallen zunächst gleichzeitig mit dem metallischen Quecksilber in flockiger Form aus, gehen jedoch rasch in kolloidaler Form in Lösung, während das kolloid verteilte Quecksilber nach einiger Zeit sich in sehr fein verteilter Form abzuscheiden beginnt.

Beim Mischen von Sulfoxylatsalvarsan mit Sublimat sind den Wirkungen von Neosalvarsan und Sublimat analoge Verhältnisse anzunehmen.

Wie zuerst Rothmann und in voller Bestätigung desselben Binz und Bauer gezeigt haben, erscheint das fein verteilte metallische Quecksilber, das durch volle Reduktion des Sublimats entsteht, als wichtigstes Reaktionsprodukt der Mischung Salvarsan-Sublimat, während die übrigen Reaktionsprodukte, namentlich auch die Arsinoxyde, nicht in wesentlichen Mengen auftreten.

Die Einführung von Neosalvarsan-Sublimatgemischen nach Linser bedeutet also eine Zuführung von metallischem Quecksilber in höchstem Dispersitätsgrade, in einer zum Teil, jedoch nicht in erheblichem Maße durch das beigefügte Sublimat veränderten Neosalvarsanlösung, die ihrerseits kolloid ist.

Man kann die bei Mischung von Neosalvarsan und Sublimat in dem von Li inser angegebenen Verhältnis vorgehenden chemischen Vorgänge folgendermaßen präzisieren (siehe S. 108).

Diese, bei Mischung von Sublimat und Salvarsan auftretenden kolloidalen Phänome sind für die akuten Giftwirkungen der Hg-Verbindungen von Bedeutung. Wie aus den von mir in Gemeinschaft mit Frl. Le u pold ausgeführten Versuchen, von denen ich hier einige Beispiele gebe, hervorgeht, wird das Sublimat z. B. durch die Beimischung kleiner Mengen von Neosalvarsan oder von Sulfoxylatsalvarsan (Präparat Nr. 1882) entgiftet. 
20 Gewichtsteile Neosalvarsan +1 Gewichtsteil Sublimat entsprechend 70 Molekülen $"+9$ Molekülen $"$

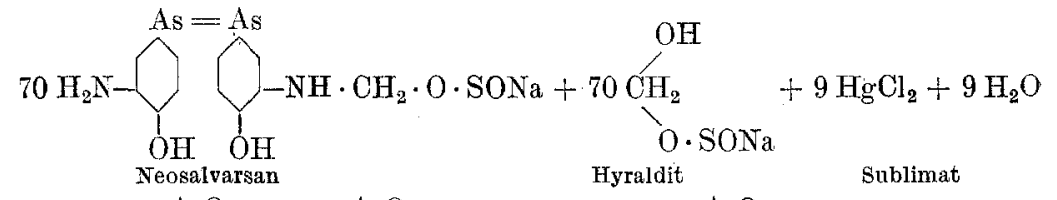

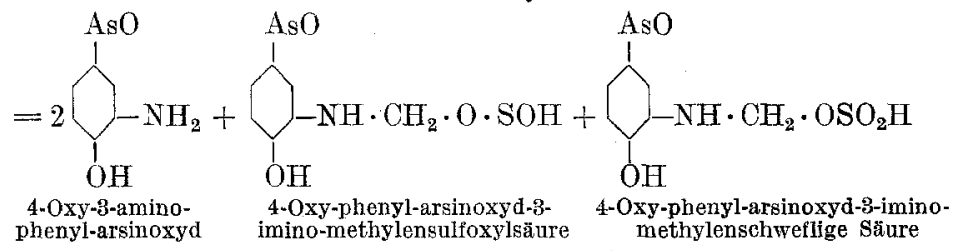

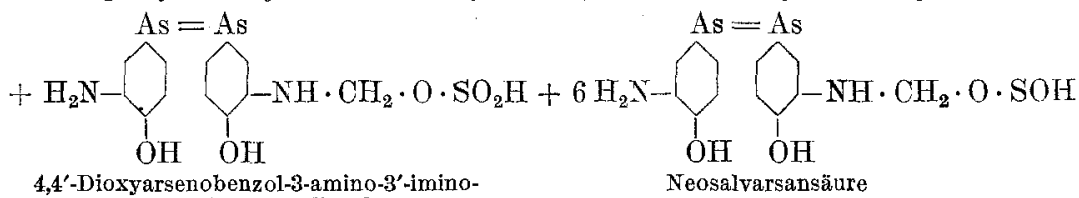

4,4'-Dioxyarsenobenzol-3-amino-3'-iminomethylenschweflige säure

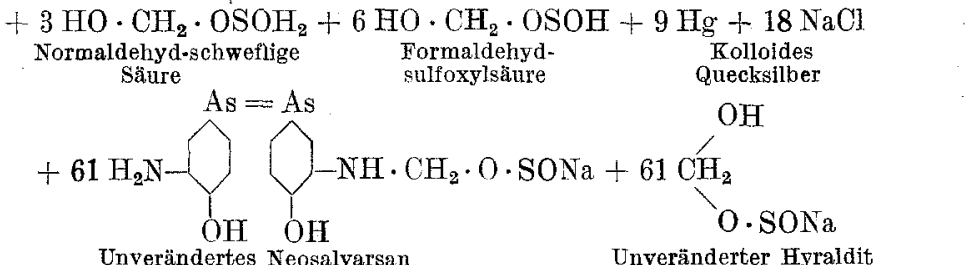

Auf die Entstehung einer erheblichen Menge von kolloidalem Quecksilber kann diese Entgiftung nicht zurückgeführt werden. Denn die Giftigkeit des kolloidalen $\mathrm{Hg}$ entspricht annähernd derjenigen des $\mathrm{HgCl}_{2}$. Es ist vielmehr wahrscheinlich, daß durch innige Mischung des entstehenden kolloidalen $\mathrm{Hg}$ mit dem Salvarsan letzteres wie eine Art Schutzkolloid für das Quecksilber wirkt, zugleich die direkte Protoplasmagiftwirkung des $\mathrm{Hg}$ auf die Zellen oder die Verankerung an die Organe (Organotropie) verändernd. Umgekehrt werden die genannten Salvarsanpräparate durch das Sublimat nicht wesentlich in ihrer Giftig. keit für Mäuse gesteigert; erst bei länger dauernder Einwirkung tritt die akute, hauptsächlich auf Bildung von Arsinoxyden beruhende Oxydationswirkung des Sublimats zutage. Die Quecksilbervergiftung der Mäuse vollzieht sich bei Verwendung der kleinen Dosen, an denen allein die Entgiftung genauer studiert und kontrolliert werden kann, nicht akut. Die Salvarsanpräparate aber wirken um so stärker, je mehr sie oxydiert sind. Daher die Unterschiede, die auf den Tabellen in den einzelnen Kolumnen bezüglich der Zeitdauer des Todeseintritts zutage treten. Bei der Auswertung der Salvarsanpräparate (Neosalvarsan, Silbersalvarsan, Neosilbersalvarsan und Sulfoxylatsalvarsan Nr. 1882) 
gemischt mit Novasurol und Sublimat, bei syphilitischen Kaninchen lassen sich einige bemerkenswerte Tatsachen verzeichnen (Tab. VII, VIII, IX).

Wir müssen auf diese Phänomene vom chemotherapeutischen Standpunkte etwas eingehen. Durch die Beimischung kleiner Dosen von Sublimat oder Novasurol zum Salvarsan tritt keine Erhöhung der Giftigkeit der Salvarsanpräparate ein, wohl aber eine Verringerung der akut auf die Spirochäten wirkenden Dosis. Es wird ferner ein schnelleres Verschwinden der Spirochäten nach Einverleibung der Gemische erzielt, als es bei gleichen Dosen oder sogar noch höheren Dosen der genannten Salvarsanpräparate ohne Hg erfolgt. Die Schanker werden rascher weich als bei gleichen oder höheren Dosen Salvarsan ohne $\mathrm{Hg}$, die Ödeme gehen zunächst zurück. Wir haben also eine chemotherapeutische Aktivierung der Salvarsanwirkung auf die Spirochäten vor uns, die nicht auf die Entstehung kleiner Mengen von Arsinoxyden bei der Mischung zurückgeführt werden kann.

Da das Novasurol und das Sublimat ohne Salvarsan erst in solchen Dosen auf die Syphilisspirochäten im Kaninchenkörper wirken, die nahe an der verträglichen Dosis liegen oder mit ihr zusammenfallen, und da ein Ausheilen der syphilitischen Primäraffekte selbst dann bei den meisten Tieren nicht erzielt wird, weil sie infolge der relativ hohen Quecksilberdosis sterben, so kann die Beimischung der kleinen Menge von Quecksilber nicht ohne weiteres im Sinne einer einfachen Kombinationswirkung und dadurch erfolgenden Potenzierung der Wirkung gedeutet werden. Die für Narcotica (Bürgi) sowie für Chemotherapeutica und Desinfektionsmittel angenommene Hypothese der verschiedenen Angriffspunkte mehrerer Medikamente (Ehrlich, Bürgi) kann zur Erklärung dieser potenzierten Effekte nicht herangezogen werden. Wir müssen vielmehr nach anderen Erklärungen suchen. Da ist zunächst zu bemerken, daß die Dauerwirkung, $d$. h. die rezidivfreie Wirkung der Präparate durch die kleine Beimengung von Quecksilberpräparaten trotz schnellen Verschwindens der Spirochäten nicht gewährleistet wird. Es treten trotz raschen Verschwindens der Spirochäten und langsamem Rückgang der Schanker bis zu kleinsten Infiltraten (,fast glatt") auffallend häufig und früh Reindurationen mit Spirochäten ein. Das deutet darauf hin, daß wir nicht eine durch Salvarsan verstärkte direkte Wirkung der Hg-Präparate auf die Spirochäten annehmen können. Man kann verschiedene Erklärungen für die geschilderte chemotherapeutische Aktivierung heranziehen: am wahrscheinlichsten ist, daß durch die stark an das syphilitische Gewebe und an die Spirochäten verankerten Salvarsanpräparate das in feinster Verteilung und zum Teil kolloidal ausgefällte $\mathrm{Hg}$, für das die auch auf diese Weise stärker kolloid gewordenen Salvarsanpräparate gewissermaßen ein Schutz- 
110 W. Kolle; Über die chemotherapeutische Aktivierung der

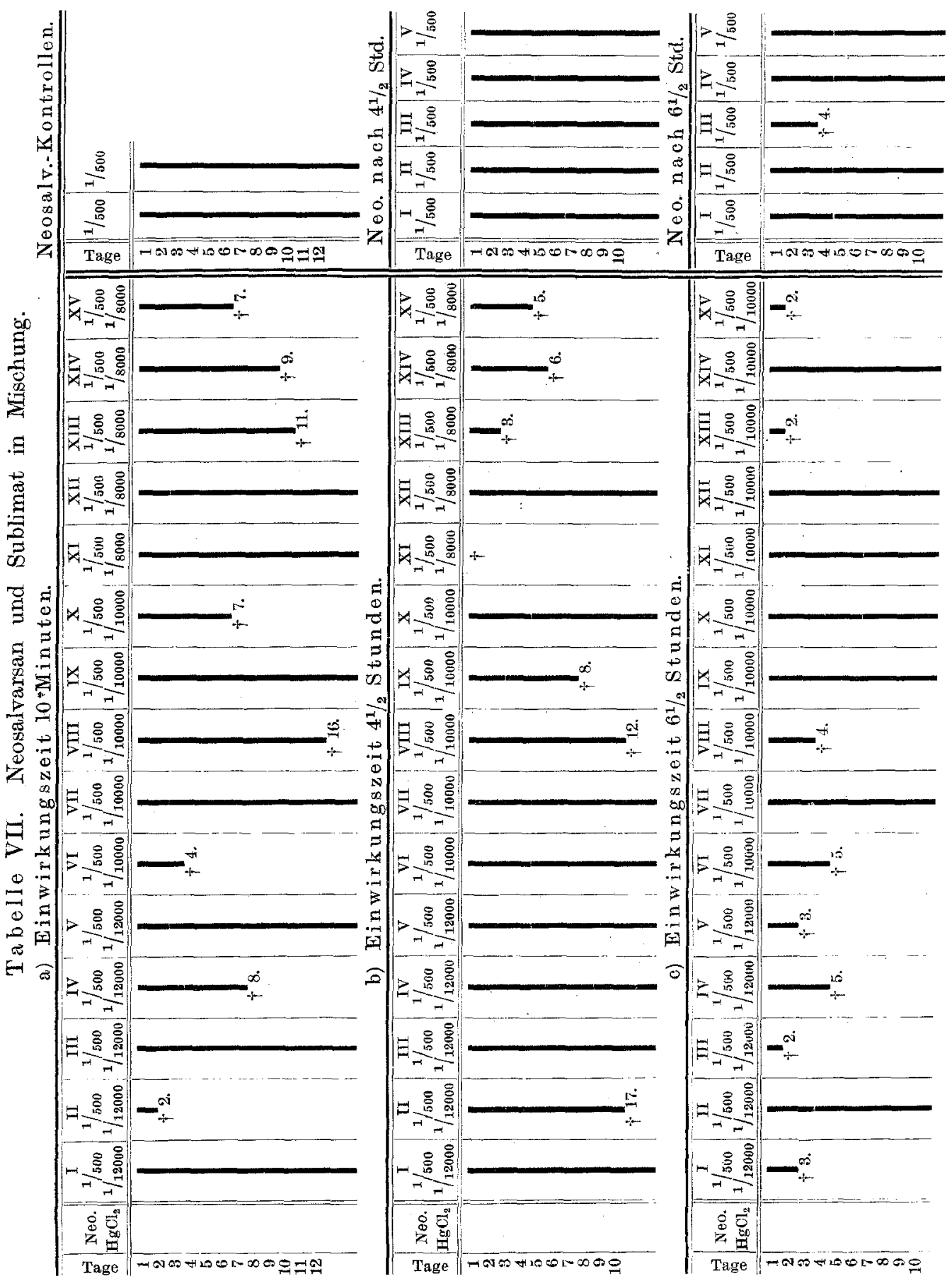


Salvarsanpräparate auf Grund von Versuchen bei experim. Kaninghensyphilis. 111

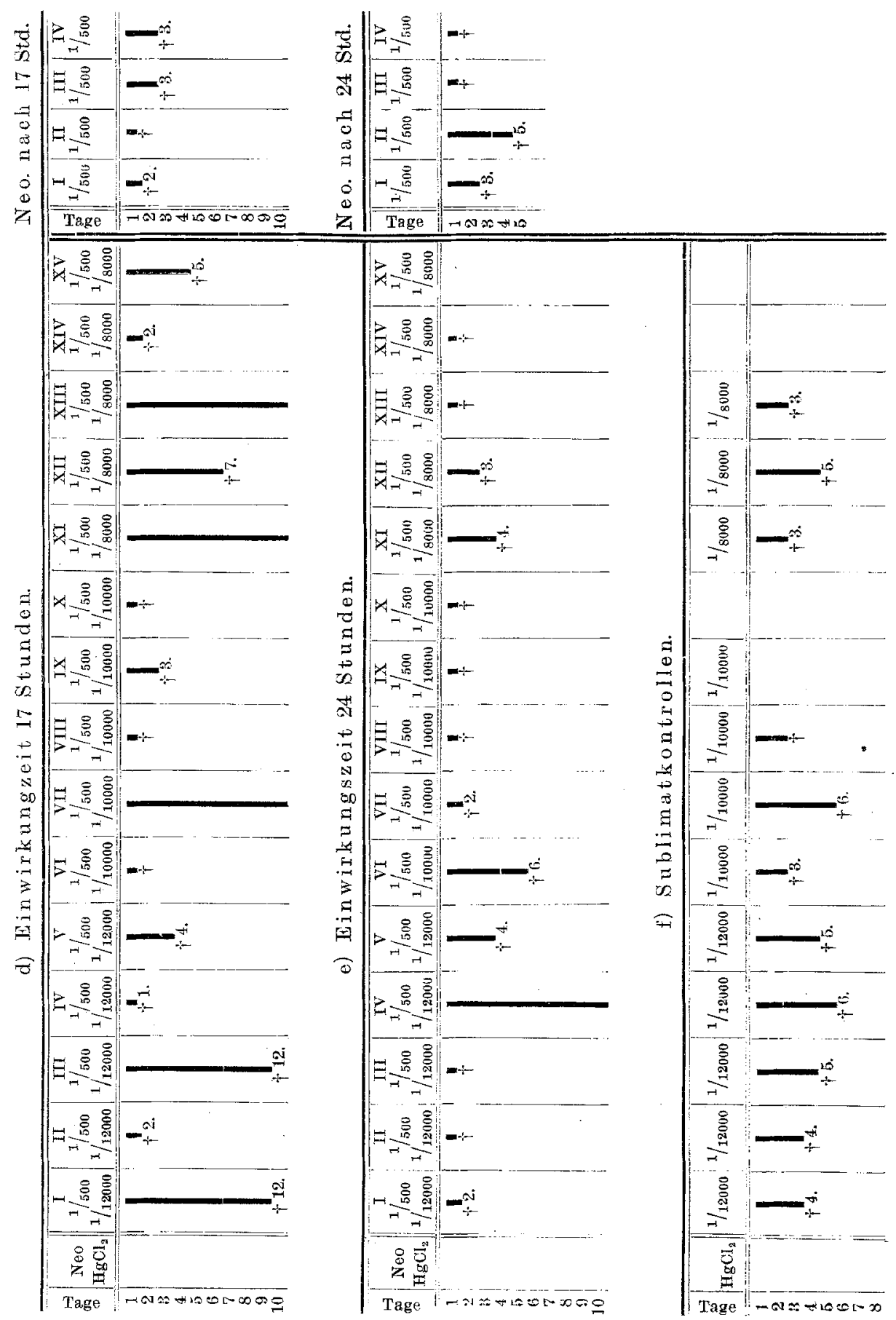




\begin{tabular}{|c|c|c|c|c|c|c|}
\hline \multirow{2}{*}{$\begin{array}{l}\text { Fanin- } \\
\text { chan- } \\
\text { Nr. }\end{array}$} & \multirow{2}{*}{$\begin{array}{l}\text { Durchmesser } \\
\text { der Schanker } \\
\text { Spirochäten } \\
\text { vor Injektion }\end{array}$} & \multirow[b]{2}{*}{ Dosis pro Kilo } & \multicolumn{2}{|c|}{ 1. Tag } & \multicolumn{2}{|c|}{ 2. Tag } \\
\hline & & & $\begin{array}{l}\text { Spirochäten- } \\
\text { befund }\end{array}$ & $\begin{array}{l}\text { Schanker- } \\
\text { gröbe }\end{array}$ & $\begin{array}{l}\text { Spirochäten- } \\
\text { befund }\end{array}$ & $\begin{array}{l}\text { Schanker- } \\
\text { größe }\end{array}$ \\
\hline 650 & $\begin{array}{l}\text { I. } 1,7 \times 1,8 \times 1,3 \\
\text { L. }+++ \text { g. bew. } \\
\text { L. } 1,4 \times 1,8 \times 1,1\end{array}$ & 0,02 & + s. w. unbew. & $1,5 \times 1,8 \times 1,2$ & 0 & - \\
\hline 649 & $\begin{array}{l}\text { L. } 1,4 \times 1,8 \times 1,1 \\
\text { R. } 1,5 \times 1,8 \times 1,1 \\
\text { L. }+++ \text { g. bew. } \\
\text { R. }+++ \text { g. bew. }\end{array}$ & 0,015 & $\begin{array}{c}\text { R. }+ \text { s. w. unbew. } \\
\text { L. + s. w. } \\
\text { z. T. unbew. }\end{array}$ & $\begin{array}{l}1,3 \times 1,8 \times 1,2 \\
1,5 \times 1,8 \times 1,2\end{array}$ & $\begin{array}{l}0 \\
0\end{array}$ & - \\
\hline 646 & $\begin{array}{l}\text { R. } 1,5 \times 1,8 \times 1,2 \\
\text { L. }+++ \text { g. bew. }\end{array}$ & 6,01 & $t+\mathrm{g}$. bew. & $1,5 \times 1,8 \times 1,1$ & + g. bew & $1,5 \times 1,6 \times 1,1$ \\
\hline 1118 & $\begin{array}{l}\text { L. } 1,7 \times 1,9 \times 1,4 \\
\text { L. }++ \text { g. bew. }\end{array}$ & 0,009 & + s. w. unbew. & $1,6 \times 1,8 \times 1,3$ & 0 & $1,6 \times 1,7 \times 1,3$ \\
\hline 868 & $\begin{array}{l}\text { R. } 2,3 \times 2,4 \times 1,2 \\
\text { R. }+++ \text { g. bew. }\end{array}$ & 0,008 & + wew. & $2,2 \times 2,4 \times 1,2$ & 0 & $2,0 \times 2,4 \times 1,1$ \\
\hline 1131 & $\begin{array}{l}\text { R. } 2,0 \times 2,2 \times 1,3 \\
\text { L. } 1,7 \times 1,7 \times 1,2 \\
\text { R. } t+\text { g. bew. } \\
\text { L. } t++ \text { g. bew. }\end{array}$ & 0,007 & $\begin{array}{l}++ \text { bew. } \\
+\quad \text { w. g. bew. }\end{array}$ & $\begin{array}{l}1,8 \times 1,8 \times 1,3 \\
1,7 \times 1,7 \times 1,2\end{array}$ & $\begin{array}{l}+ \text { s. w. bew. } \\
+ \text { bew. }\end{array}$ & $\begin{array}{l}1,7 \times 1,9 \times 1,2 \\
1,6 \times 1,6 \times 1,2\end{array}$ \\
\hline 1108 & $\begin{array}{l}\text { R. } 1,6 \times 1,6 \times 1,0 \\
\text { L. } 1,6 \times 2,0 \times 1,2 \\
\text { R. }++ \text { g. bew. } \\
\text { L. + + g. bew. }\end{array}$ & 0,006 & $\begin{array}{l}\text { R. + bew. } \\
\text { L. + unb. }\end{array}$ & $\begin{array}{l}1,7 \times 1,5 \times 1,0 \\
1,5 \times 1,2 \times 1,2\end{array}$ & $\begin{array}{l}+ \text { s. w. unb. } \\
+ \text { s. w. unb. }\end{array}$ & $\begin{array}{l}1,6 \times 1,5 \times 1,1 \\
1,4 \times 1,4 \times 1,1\end{array}$ \\
\hline
\end{tabular}

Dosis tax. 0,02 Dosis tox. 0,15

posis tol. 0,015 Dosis tol. 0,13

Tabelle IX. Wirkung des, Neosilbersalvarsan und

\begin{tabular}{|c|c|c|c|c|c|c|}
\hline \multirow{2}{*}{$\begin{array}{c}\text { Eanin- } \\
\text { chen- } \\
\text { Nr. }\end{array}$} & \multirow{2}{*}{$\begin{array}{c}\text { Durchmesser } \\
\text { der Schanker } \\
\text { Spirochäten } \\
\text { vor Injektion } \\
\end{array}$} & \multirow{2}{*}{ Dosis pro Kilo } & \multicolumn{2}{|c|}{ 1. Tag } & \multicolumn{2}{|c|}{ 2. Tag } \\
\hline & & & $\begin{array}{c}\text { Spirochäten- } \\
\text { befund }\end{array}$ & $\begin{array}{l}\text { Schanker- } \\
\text { durchmesser }\end{array}$ & $\begin{array}{c}\text { Spirochäten- } \\
\text { befund }\end{array}$ & $\begin{array}{c}\text { Schanker- } \\
\text { durchmesser }\end{array}$ \\
\hline 1028 & 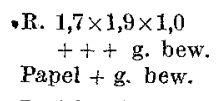 & $\begin{array}{l}0,01 \\
0,003\end{array}$ & $\begin{array}{l}\text { R. + w. unb. } \\
\text { P. + s. w. unb. }\end{array}$ & $1,7 \times 2,0 \times 1,0$ & $\begin{array}{l}0 \\
0\end{array}$ & $1,5 \times 1.8 \times 1,1$ \\
\hline 1073 & $\begin{array}{l}\text { R. } 1,6 \times 1,5 \times 1,1 \\
\text { L. } 1,3 \times 1,6 \times 1,0 \\
\text { R. }+++ \text { g. bew. } \\
\text { L. }+++ \text { g. bew. }\end{array}$ & $\begin{array}{l}0,008 \\
0,003\end{array}$ & $\begin{array}{l}\text { R. + w. unb. } \\
\text { L. + w. unb. }\end{array}$ & $\begin{array}{l}\mathbf{1 , 5} \times 1,5 \times 1,1 \\
1,4 \times 1,5 \times 1,0\end{array}$ & $\begin{array}{c}0 \\
+ \text { s. } w . \text { unb. }\end{array}$ & $\begin{array}{l}1,3 \times 1,4 \times 1,9 \\
1,3 \times 1,5 \times 1,01\end{array}$ \\
\hline 1184 & $\begin{array}{l}\text { R. } 1,7 \times 1,9 \times 1,2 \\
\text { L. } 1,7 \times 1,8 \times 1,2 \\
\text { R. }+++ \text { g. bew. } \\
\text { L. }++ \text { g. bew. }\end{array}$ & $\begin{array}{l}0,007 \\
0,001\end{array}$ & $\begin{array}{l}\text { B. }+u n b . \\
\text { L. }+u m b\end{array}$ & $\begin{array}{l}1,8 \times 1,9 \times 1,2 \\
1,6 \times 1,9 \times 1,3\end{array}$ & $\begin{array}{l}+ \text { w. unb. } \\
+ \text { w. unb. }\end{array}$ & $\begin{array}{l}1,7 \times 1,8 \times 1,1 \\
1,5 \times 1,8 \times 1,2\end{array}$ \\
\hline 1182 & $\begin{array}{l}\text { R. } 1,5 \times 1,7 \times 1,0 \\
\text { L. } 1,8 \times 2,0 \times 1,2 \\
\text { R. }+++ \text { g. bew. } \\
\text { L. }++ \text { g. bew. }\end{array}$ & $\begin{array}{l}0,006 \\
0,004\end{array}$ & $\begin{array}{l}\text { R. + + unb. } \\
\text { L. + + unb. }\end{array}$ & $\begin{array}{l}1,6 \times 1,8 \times 1,0 \\
1,8 \times 2,0 \times 1,2\end{array}$ & $\begin{array}{l}+ \text { anb. } \\
+ \text { w. unb. }\end{array}$ & $\begin{array}{l}1,5 \times 1,6 \times 0,9 \\
1,7 \times 1,8 \times 1,2\end{array}$ \\
\hline 1134 & $\begin{array}{l}\text { R. } 1,7 \times 2,2 \times 1,8 \\
\text { R. }++ \text { g. bew. }\end{array}$ & $\begin{array}{l}0,004 \\
0,005\end{array}$ & + bew. & $2,0 \times 2,3 \times 1,5$ & + w. bew. & $2,0 \times 2,8 \times 1,5$ \\
\hline 1109 & $\begin{array}{l}\text { R. } 1,7 \times 1,8 \times 1,0 \\
\text { L. } 2,0 \times 2,1 \times 1,1 . \\
\text { R. }+++ \text { g. bew. } \\
\text { L. } \quad+\text { g. bew. }\end{array}$ & $\begin{array}{l}0,001 \\
0,008\end{array}$ & $\begin{array}{l}\text { R. + bew. } \\
\text { L. + w. bew. }\end{array}$ & $\begin{array}{l}1,6 \times 1,8 \times 1,0 \\
1,9 \times 2.0 \times 1,1\end{array}$ & $\begin{array}{l}+ \text { w. bew. } \\
+ \text { s. w. bew. }\end{array}$ & $\begin{array}{l}1,5 \times 1,6 \times 1.0 \\
1,8 \times 1,9 \times 1,0\end{array}$ \\
\hline 831 & $\begin{array}{l}\text { R. } 2,8 \times 2,7 \times 1,1 \\
\text { L. } 2,7 \times 2,8 \times 0,9 \\
\text { R. } \quad+\text { "w. g. bew. } \\
\text { L. ++ g. bew. }\end{array}$ & $\begin{array}{l}0,003 \\
0,005\end{array}$ & $\begin{array}{l}\text { R. } 0 \\
\text { L. + bew. }\end{array}$ & $\begin{array}{l}2,7 \times 2,8 \times 1,2 \\
2,7 \times 2,9 \times 1,0\end{array}$ & $\begin{array}{l}+ \text { w. g. bew. } \\
+ \text { w. g. bew. }\end{array}$ & $\begin{array}{l}2.5 \times 2,7 \times 1,2 \\
2,7 \times 2,7 \times 1,0\end{array}$ \\
\hline 1072 & $\begin{array}{l}\text { R. } 2,0 \times 2,0 \times 1,2 \\
\text { L. } 2,0 \times 2,1 \times 1,5 \\
\text { R. }+++ \text { g. bew. } \\
\mathbf{L}_{.}+++ \text {g. bew. }\end{array}$ & $\begin{array}{l}0,002 \\
0,003\end{array}$ & $\begin{array}{l}\text { R. }+++ \text { g. b. } \\
\text { L. }+++ \text { g. b. }\end{array}$ & $\begin{array}{l}2,0 \times 2,1 \times 1,3 \\
2,0 \times 2,1 \times 1,3\end{array}$ & $\begin{array}{l}++ \text { bew. } \\
++ \text { g. bew. }\end{array}$ & $\begin{array}{l}1,9 \times 2,1 \times 1,3 \\
2,0 \times 2,1 \times 1,4\end{array}$ \\
\hline
\end{tabular}


Salvarsanpräparate auf Grund von Versuchen bei experim. Kaninchensyphilis. 113 auf den Syphilisschanker am Kaninchenhoden.

\begin{tabular}{|c|c|c|c|c|c|c|}
\hline \multicolumn{2}{|c|}{ 3. Tag } & \multicolumn{2}{|c|}{ 4. Tag } & \multicolumn{2}{|c|}{ 5. Tag } & \\
\hline $\begin{array}{l}\text { Spirochäten- } \\
\text { befund }\end{array}$ & $\begin{array}{c}\text { Schankex- } \\
\text { größe }\end{array}$ & $\begin{array}{l}\text { Spirochäten- } \\
\text { befund }\end{array}$ & $\begin{array}{l}\text { Schanker- } \\
\text { grôße }\end{array}$ & $\begin{array}{l}\text { Spirochäten- } \\
\text { befund }\end{array}$ & $\begin{array}{l}\text { Schanker- } \\
\text { gröBe }\end{array}$ & \\
\hline 0 & $1,4 \times 1,4 \times 1,0$ & 0 & $1,3 \times 1,4 \times 1,0$ & 0 & $1,2 \times 1,3 \times 1,0$ & am 21. Tag glatt \\
\hline 0 & $1,2 \times 1,4 \times 1,0$ & 0 & $1,2 \times 1, \overrightarrow{3} \times 1,0$ & 0 & $1,0 \times 1,2 \times 1,0$ & \\
\hline 0 & $1,1 \times 1,3 \times 0,9$ & 0 & $1,1 \times 1,3 \times 0,9$ & 0 & $1,1 \times 1,2 \times 0,8$ & am 21. Tag glatt \\
\hline t s. w. bew. & $1,3 \times 1 ; 5 \times 1,1$ & + w. bew. & $1,2 \times 1,4 \times 1,0$ & & & $\begin{array}{c}\text { am 31. Tag R. glatt, } \\
\text { L. fast glatt }\end{array}$ \\
\hline 0 & $1,5 \times 1,6 \times 1,0$ & 0 & $1,3 \times 1,5 \times 1,1$ & 0 & $1,3 \times 1,6 \times 0,9$ & glatt \\
\hline 0 & $1,9 \times 2,3 \times 1,1$ & 0 & $1,9 \times 2.0 \times 1,0$ & 0 & $1,7 \times 1,7 \times 1,0$ & $\begin{array}{c}\text { am 28. Tag } \\
\text { Sp. }+\end{array}$ \\
\hline $\begin{array}{l}0 \\
0\end{array}$ & $\begin{array}{l}1,6 \times 2,0 \times 1,1 \\
1,2 \times 1,5 \times 0,9\end{array}$ & $\begin{array}{c}+ \text { s. w. bew. } \\
0\end{array}$ & $\begin{array}{l}1,6 \times 2,0 \times 1,1 \\
1,3 \times 1,3 \times 0,8\end{array}$ & $\begin{array}{c}0 \\
+\quad \text { w. bew. }\end{array}$ & $\begin{array}{l}1,6 \times 2,1 \times 0,9 \\
1,4 \times 1,4 \times 0,9\end{array}$ & nicht ausgeheilt \\
\hline & & & & & & \\
\hline 0 & $1,5 \times 1,5 \times 1,0$ & 0 & $1, \overline{\mathbf{b}} \times 1,4 \times 1,1$ & + w. unb. & $1,5 \times 1,5 \times 1,0$ & am 22. $\mathrm{Tag}$ \\
\hline 0 & $1,4 \times 1,5 \times 1,0$ & 0 & $1,4 \times 1,5 \times 1,0$ & 0 & $1,4 \times 1,5 \times 1,0$ & $\mathrm{Sp} .+$ \\
\hline
\end{tabular}

Novasurol:" auf den Syphilisschanker am Kaninchenhoden.

\begin{tabular}{|c|c|c|c|c|c|c|}
\hline \multicolumn{2}{|c|}{ 3. Tag } & \multicolumn{2}{|c|}{ 4. Tag } & \multicolumn{2}{|c|}{ 5. Tag } & \\
\hline $\begin{array}{l}\text { Spirochäten- } \\
\text { befund }\end{array}$ & $\begin{array}{l}\text { Schanker- } \\
\text { durchmesser }\end{array}$ & $\begin{array}{l}\text { Spirochäten- } \\
\text { befund }\end{array}$ & $\begin{array}{l}\text { Schanker- } \\
\text { durchmesser }\end{array}$ & $\begin{array}{l}\text { Spirochäten- } \\
\text { befund }\end{array}$ & $\begin{array}{l}\text { Schanker- } \\
\text { durchmesser }\end{array}$ & \\
\hline $\begin{array}{l}0 \\
0\end{array}$ & $1,5 \times 1,6 \times 1,0$ & $\begin{array}{l}0 \\
0\end{array}$ & $1,5 \times 1,5 \times 0,8$ & $\begin{array}{l}0 \\
0\end{array}$ & $1,4 \times 1,4 \times 0,8$ & am 15. Tag glatt \\
\hline $\begin{array}{c}\theta \\
+ \text { s. w. unb. }\end{array}$ & $\begin{array}{l}1,2 \times 1,3 \times 0,9 \\
1,2 \times 1,4 \times 1,0\end{array}$ & $\begin{array}{l}0 \\
0\end{array}$ & $\begin{array}{l}1,2 \times 1,2 \times 1,0 \\
1,2 \times 1,3 \times 0,9\end{array}$ & $\begin{array}{l}0 \\
0\end{array}$ & $\begin{array}{l}1,2 \times 1,2 \times 0,8 \\
1,0 \times 1,2 \times 0,7\end{array}$ & am 30. Tag glatt \\
\hline $\begin{array}{l}0 \\
0\end{array}$ & $\begin{array}{l}1 ; 6 \times 1,6 \times 1,0 \\
1,5 \times 1,6 \times 1,0\end{array}$ & $\begin{array}{l}0 \\
0\end{array}$ & $\begin{array}{l}1,5 \times 1,5 \times 0,8 \\
1,4 \times 1,5 \times 1,0\end{array}$ & $\begin{array}{l}0 \\
0\end{array}$ & $\begin{array}{l}1,5 \times 1,5 \times 0,8 \\
1,8 \times 1,4 \times 1,0\end{array}$ & am 22. Tag glatt \\
\hline $\begin{array}{l}+ \text { s. w. unb. } \\
\text { T s. w. unb. }\end{array}$ & $\begin{array}{l}1,8 \times 1,4 \times 0,7 \\
1,5 \times 1,6 \times 1,0\end{array}$ & $\begin{array}{l}0 \\
0\end{array}$ & $\begin{array}{l}1,3 \times 1,3 \times 0,7 \\
1,4 \times 1,6 \times 1,0\end{array}$ & $\begin{array}{l}0 \\
0\end{array}$ & $\begin{array}{l}1,2 \times 1,1 \times 0,6 \\
1,3 \times 1,4 \times 0,9\end{array}$ & am 14. Tag Spir. + \\
\hline T w. unb. & $2,0 \times 2,2 \times 1,2$ & + g. bew. & $2,0 \times 2,2 \times 1,2$ & + g. bew. & $2,3 \times 2,5 \times 1,3$ & nicht ausgeheilt \\
\hline $\begin{array}{l}\text { T s. w. unb. } \\
T \text { s. w. unb. }\end{array}$ & $\begin{array}{l}1,5 \times 1,6 \times 0,9 \\
1,6 \times 1,9 \times 1,0\end{array}$ & $\begin{array}{l}0 \\
0\end{array}$ & $\begin{array}{l}1,5 \times 1,6 \times 1,0 \\
1,7 \times 1,8 \times 1,0\end{array}$ & $\begin{array}{l}0 \\
0\end{array}$ & $\begin{array}{l}1,4 \times 1,5 \times 0,9 \\
1,7 \times 1,7 \times 1,0\end{array}$ & am 33. Tag Spir. + \\
\hline- & - & - & - & $\begin{array}{l}0 \\
0\end{array}$ & $\begin{array}{l}2,0 \times 2,5 \times 1,0 \\
2,2 \times 2,1 \times 1,1\end{array}$ & am 10. Tag Spir. + \\
\hline $\begin{array}{l}+ \text { g. bew. } \\
+\div \text { g. bew. }\end{array}$ & $\begin{array}{l}1,7 \times 2,0 \times 1,3 \\
1,8 \times 2,0 \times 1,2\end{array}$ & $\begin{array}{l}++ \text { g. bew. } \\
++ \text { g. bew. }\end{array}$ & $\begin{array}{l}1,8 \times 2,1 \times 1,3 \\
1,9 \times 2,0 \times 1,3\end{array}$ & $\begin{array}{l}++ \text { g. bew. } \\
++ \text { g. bew. }\end{array}$ & $\begin{array}{l}2,0 \times 2,2 \times 1,3 \\
2,0 \times 2,1 \times 1,4\end{array}$ & nicht ausgelleilt \\
\hline
\end{tabular}


kolloid darstellen, mit in die syphilitischen Gewebe hineingerissen wird und dort indirekt auf die Spirochäten wirkt. Es ist da zu denken an die entwicklungshemmende Wirkung der $\mathrm{Hg}$-Eiweißverbindungen, die entstehen. Alle $\mathrm{Hg}$-Verbindungen, mögen sie in wasserlöslicher oder wasserunlöslicher Form einverleibt sein, werden im Organismus in gelöste Form ${ }_{3}$ und durch Blut und Lymphe in alle Zellen des Körpers übergeführt. Sie werden so allgemeine Protoplasmagifte, zugleich aber auch entwicklungshemmend für die Vermehrung der Spirochäten. Bereits Ehrlich hatte diese Art der Sterilisierung durch Verhinderung der Vermehrungsfähigkeit der Spirochäten ins Auge gefaßt. Die entwicklungshemmende Wirkung der $\mathrm{Hg}$-Verbindungen kann man sich aber auch so erklären, daß man sagt: $\mathrm{Hg}$ verändert die Zellen biologisch und macht sie zu Nährböden für die Spirochäten ungeeignet bzw. führt zur Ausstoßung der für die Spirochäten nötigen Nährstoffe, so daß die Spirochäten nicht in die Zellen eindringen oder auf ihnen leben können. Ich erinnere in dieser Beziehung nur an die Vorstellungen, die bezüglich der WaR. und ihrer Bedeutung für die Entstehung der Spirochätenverbreitung im Organismus neuerdings von Wasser mann vorgebracht worden sind, ohne daß ich hier zu den theoretischen Vorstellungen auf Grund von Versuchen bisher Stellung nehmen konnte. Daneben kommt aber die zellenaktivierende Wirkung der Quecksilberpräparate in Frage. Das Hg wirkt auf die Zellen aktivierend und führt zu einer Mobilisierung spirochätenfeindlicher Stoffe. Man kann das als Umstimmung oder Aktivierung der Gewebe bezeichnen und sie in Analogie setzen zu der sogenannten Protoplasmaaktivierung.

Bei dieser Sachlage ist es wohl am besten, von einer neuen Hypothese abzusehen, vielmehr einfach die Tatsache zu registrieren, daß die $\mathrm{Hg}$ Präparate auf die Salvarsanpräparate aktivierend wirken, und zwar im Sinne der Erhöhung der akuten chemotherapeutischen Wirkung der Arsenobenzolderivate auch in kleinen Dosen. Ich möchte deshalb auch von einer chemotherapeutischen Aktivierung sprechen, ohne daß wir imstande wären, das Wesen dieses Vorgangs, soweit es die Heilung der Primäraffekte der Kaninchensyphilis betrifft, völlig zu überschauen. Sicher ist nur, daß die Hg-Verbindungen, gemischt mit den Salvarsanpräparaten, die starke Affinität zu den Spirochäten und zum syphilitischen Gewebe haben, in die spirochätenhaltigen Teile des Organismus, also hier hauptsächlich in die Schanker, in viel größerem Umfange gelangen, als wenn Hg-Verbindungen allein injiziert werden.

Wichtig ist noch, daß man bei Rekurrensinfektion, bei der die Anwendung von Mischungen des Novasurols und des Sublimats mit verschiedenen Salvarsanpräparaten versucht wurde, keine Erhöhung des Titers der Salvarsanpräparate durch $\mathrm{Hg}$ her- 
beiführt. Bei Rekurrens tritt ja auch ohne Salvarsan schon eine krisenhafte, auf Entstehung von Antikörpern beruhende Sterilisierung des infizierten Körpers ein. Bei Anwendung von Salvarsan summiert sich diese Wirkung der Antikörper mit der direkten des Salvarsans, wird vielleicht schon ad maximum erhöht. Die Hg-Präparate haben keine direkten Angriffspunkte auf Rekurrensspirochäten. Daher findet auch keine Erhöhung der chemotherapeutischen Wirkung der Salvarsanpräparate durch $\mathrm{Hg}$ bei Rekurrens statt. Ferner sind die Rekurrensspirochäten reine Blutparasiten, die Protoplasmawirkungen der $\mathrm{Hg}$ Präparate können daher hier keine Rolle spielen.

Aus alledem ergibt sich auch, wie verschieden die Wirkung der $\mathrm{Hg}$-Salvarsangemische sowie der Quecksilbersalvarsankombinationen, bei denen $\mathrm{Hg}$ gleichzeitig mit Salvarsanpräparaten, aber an anderen Stellen einverleibt wird, sich gestaltet, verglichen mit der Wirkung der echten Metallsalvarsane. Bei diesen liegt eine einheitliche chemische Verbindung vor, nicht aber bei den Salvarsanquecksilbergemischen. Bei den Metallsalvarsanen sind nicht zwei Körper, das Salvarsan und die Metalle nebeneinander vorhanden und in chemische Wechselwirkung getreten, wobei die am Beispiel des Neosalvarsans und Sublimats näher studierten Körper auftreten können, denn es verhalten sich fast alle Quecksilberverbindungen, die organischen und anorganischen, hier prinzipiell gleich, sondern die Metallsalvarsane sind stabile, neue Verbindungen mit neuen chemischen Eigenschaften. Es erfolgen keinerlei chemische Umsetzungen mehr. Es entsteht $\mathrm{z}$. B. weder in vitro noch in vivo beim Silbersalvarsan etwa neben dem Salvarsan kolloidales Silber, sondern das Silber ist an das ganze Salvarsanmolekül, vorwiegend an die Amidogruppe, als Silber bzw. Silberoxyd gebunden. Das Silbersalvarsan entfaltet als solches seine biologischen und chemotherapeutischen Eigenschaften. Bei Kaninchensyphilis führt es auch in den kleinsten heilenden Dosen, wenn die Spirochäten rasch zum Verschwinden gebracht werden, stets zur raschen und rezidivfreien Dauerheilung der Primäraf fekte, nicht nur zu vorübergehender chemotherapeutischer Aktivierung des Salvarsans, sondern zu einer Herabsetzung der wirksamen Dosis und Hebung des chemotherapeutischen Index. Das Silber wirkt außerdem allein auch auf die Syphilisspirochäten, und zwar bei Kaninchensyphilis in therapeutiseh wirksamen Dosen. Deshalb ist das Silbersalvarsan als ein echtes Kombinationsmittel aufzufassen. Das Silber mit Salvarsan ergibt eine Potenzierung im Sinne von Ehrlich und Bürgi. Für die Quecksilbersalvarsangemische ist diese Auffassung bisher nicht möglich, vielmehr eine komplexe Wirkung im Sinne der oben gemachten Ausführungen anzunehmen. 
Von dem Gesichtspunkte der chémotherapeutischen Aktivierung der Salvarsanpräparate sind wir auch bei der Herstellung des Neosilbersalvarsans ausgegangen. Diese von mir in Gemeinschaft mit Binz und Bauer ausgeführten Arbeiten haben nach Überwindung mancher technischen Schwierigkeiten, an denen auch die Chemiker der Höchster Farbwerke Dr. Ammelburg, Dr. Scholl und Dr. Streitwolf beteiligt waren, zur Herstellung eines Präparats geführt, das gewissermaßen ein durch Metalle aktiviertes Neosalvarsan darstellt. Es ist das Neosilbersalvarsan, das chemotherapeutisch bei Kaninchensyphilis annähernd den gleichen Index hat wie das Silbersalvarsan. Es zeichnet sich aber dadurch aus, daß die Beifügung der Silberquote zum Neosalvarsan, auf deren Einzelheiten ich aus verschiedenen Gründen nicht eingehen möchte, dem neuen Präparat alle Vorteile des Neosalvarsans, namentlich die leichte Löslichkeit und leichte Verträglichkeit im Gegensatz zum Silbersalvarsan verliehen hat, unter Ausschaltung der Hauptnachteile des Neosalvarsans; der relativ schwachen Wirkung und der leichten. Oxydationsfähigkeit.

Die Lösungen von Neosilbersalvarsan sind zudem chemisch stabilisiert. Sie oxydieren an der Luft außerordentlich wenig und fallen im Gegensatz zum Silbersalvarsan auch bei längerem Stehen an der Luft nicht aus. Deshalb weisen sie auch keine Zunahme der Giftigkeit innerhalb der ersten Stunde, ja - wie die Tierversuche zeigen - nach den ersten 4 Stunden nach der Herstellung auf. Selbst nach 24stündigem Stehen an der Luft ist die Zunahme der Giftigkeit solcher Lösungen eine minimale. Ich möchte aus prinzipiellen Gründen nicht für längeres Stehenlassen der Lösungen mich aussprechen, aber innerhalb der ersten Stunde treten keinerlei Veränderungen in der Giftigkeit auf, so daß die Herstellung größerer Mengen von Lösungen für Serieninjektionen wohl möglich ist. Mit Dosen von 0,3 bis 0,5 des Neosilbersalvarsans, das wegen der in ihm enthaltenen Silberkomponente sehr nachhaltig wirkt, allein oder in gewöhnlicher Kombinationskur, lassen sich Wirkungen erzielen, wie man sie sonst mit 0,45 bis 0,60 Neosalvarsan erreicht, unter Berücksichtigung, daß der Gehalt an Arsen um 20\% geringer ist als im Neosalvarsan. In diesem Umstand dürfte ein weiterer Vorteil zu erblicken sein. Ferner läßt sich das Präparat im Gegensatz zum Silbersalvarsan leichter mit $\mathrm{Hg}$-Verbindungen mischen als Neosalvarsan.

Eine Anzahl Operationsnummern, die anfangs noch nicht ein Präparat darstellten, wie wir es unter der Bezeichnung „hyperideal ${ }^{\text {‘c }}$ gewohnt sind zu verstehen und sich deshalb nach einiger Zeit zersetzten, wiesen bei der Lösung eigenartige Trübung und unter dem Mikroskop Kugeln auf. Nach klinischer Verwendung dieser Operationsnummern 
traten in einem nicht geringen Prozentsatz Exantheme auf, die insofern interessant sind, als sie es wahrscheinlich machen, daß die - sei es prä. formierte, sei es im Körper auftretende - Ausflockung der Silbersalvarsan- und Neosilbersalvarsanlösungen mit eine der Ursachen für die Entstehung von Dermatitiden bzw. Exanthemen ist. Die technische Darstellung ist inzwischen so verbessert, daß die Proben sich dauernd, ohne eine derartige Umwandlung aufzuweisen, aufbewahren lassen.

M. H. Ich habe hier mit Rücksicht auf die Bedeutung des Quecksilbers, dieses ältesten und wie es scheint vielfach noch nicht zu entbehrenden Antisyphiliticums, über die mit diesem angestellten experimentellen Arbeiten berichtet. Die Wirkungen der Kombinationskuren, bei denen die therapeutisch verstärkten Schläge wiederholt werden, können so vielleicht erheblich wirksamer gestaltet werden. Außer den Metallen kommen für die Aktivierung der Salvarsane auch noch andere Körper als die Schwermetalle in Frage. Wir haben diese Frage seit längerer Zeit in Bearbeitung, doch sind die Versuche noch im FluB. Ich werde Ihnen später darüber berichten. Hierbei spielen vielleicht kolloidchemische Gesichtspunkte, ebenso wie die strukturchemischen Auffassungen eine Rolle. Ich will nur erwähnen, daß Phenolderivate, Körper der Chininreihe, z. B. Eucupin, gewisse Farbstoffe zu einer Aktivierung der Salvarsanpräparate führen. Ob diese Beimischung bzw. Beifügung von derartigen Körpern praktisch-therapeutische Bedeutung hat, ist klinisch zu erproben.

\section{SchluBfolgerungen.}

1. Die intravenöse Einverleibung von löslichen Quecksilberverbindungen in einem Überschuß von Salvarsan ist ungefährlicher, als erwartet werden konnte. Sie führt, wie die genaue Auswertung der Giftigkeit der Mischungen bei Kaninchen und Mäusen zeigt, zu keiner nennenswerten Giftung der Salvarsanpräparate. Durch das Salvarsan, das gewissermaßen als Schutzkolloid für das hierbei entstehende kolloidale Quecksilber dient, erfolgt im Tierversuch eine gewisse Entgiftung der mit ihm gemischen Quecksilberpräparate, was die akute Giftwirkung betrifft. In erster Linie kommen von den Salvarsanpräparaten diejenigen, welche die Formaldehydsulfoxylatgruppe enthalten, für die Mischung in Frage, da sie Angriffspunkte für die oxydierenden $\mathrm{Hg}$ Verbindungen in der Formaldehydgruppe besitzen und so zu einer $\mathrm{Ab}$ lenkung des Quecksilbers von der Arsenogruppe führen.

2. Da die Quecksilberpräparate mit den Salvarsanpräparaten in eine Wechselwirkung treten, wobei das Salvarsan zum Teil chemisch umgewandelt wird, und fast sämtlich oxydierende Wirkung haben, andererseits das Salvarsan ein starker Reduktionskörper ist, so ist, um die Arsenoverbindungen als solche möglichst intakt zu erhalten, die Verwendung von wenig oxydierenden Quecksilberverbindungen für das 
118 W. Kolle: Über d. chemotherapeutischo Aktivierung d. Salvarsanpräparate

Mischungsverfahren anzustreben. Das Sublimat erscheint in dieser Hinsicht als das am wenigsten geeignete, weil es ein sehr starkes Oxydationsmittel ist. Von den bekannten klinisch erprobten Quecksilberpräparaten dürfte das Novasurol geeigneter sein.

3. Die Wirkung aller Salvarsanpräparate kann durch Beimischung von Quecksilberverbindungen gesteigert werden, und zwar in chemotherapeutischem Sinne, soweit es die akute Wirkung auf die Spirochäten betrifft.

4. Die chemotherapeutische Aktivierung der Salvarsanpräparate durch Quecksilber - echte wasserlösliche $\mathrm{Hg}$-Salvarsane sind nicht darstellbar - kann nicht in Parallele gestellt werden zur Wirkung der Metallsalvarsane, die einheitliche wasserlösliche Körper mit neuen chemischen und chemotherapeutischen Eigenschaften darstellen. Bei den Mischungen der bisher bekannten Quecksilberverbindungen mit Salvarsanpräparaten kommt neben der Entstehung von Arsinoxyden die in ihren Einzelheiten noch unbekannte, jedenfalls auf mehreren Faktoren beruhende Wirkung des metallischen Quecksilbers in kolloidaler Form in Frage.

5. Dauerwirkung der Hg-Salvarsanmischungen, bei denen nur kleine Mengen von $\mathrm{Hg}$ - wie z. B. bei Linsers Verfahren - einverleibt werden, ist bei Kaninchensyphilis nicht vorhanden, trotz Hebung der chemotherapeutischen Wirkung der Gemische und chemotherapeutischer Aktivierung.

6. Die Kombinierung von Metallsalvarsanen mit löslichen Quecksilberverbindungen führt zu einer weiteren chemotherapeutischen Aktivierung der Salvarsanwirkung. Aussichtsreich für diese Zwecke, z. B. bei der Abortivbehandlung der Syphilis, erscheint die Mischung des Neosilbersalvarsans mit Novasurol. Sublimat.Neosalvarsangemische nach Linser sind jedenfalls nicht zuempfehlen und sollten, wenn sie überhaupt angewandt werden, unmittelbar nach der Herstellung eingespritzt werden.

7. Es ist experimentell und klinisch zu prüfen, ob diese Methode der Injektion von Salvarsan-Hg-Gemischen der gewöhnlich als Kombinationskur bezeichneten Anwendung von Salvarsan and $\mathrm{Hg}$ - wobei große Quecksilbermengen intramuskulär angewandt werden - überlegen ist. Klinisch dürfte auf Grund der Tierversuche die Frage der Leberschädigung und des Ikterus wegen der Mischung von kolloidalem Quecksilber mit dem gleichfalls kolloidalen Salvarsangemisch im Auge zu behalten sein.

Vor der Verwendung der Salvarsanquecksilbergemische in die Therapie des Praktikers dürfte eine eingehende Erprobung des Verfahrens in Kliniken notwendig sein, wie sie von Bruck für Neosalvarsan-Novasurolgemische, von Schönfeld für Silbersalvarsan-Novasurolmischungen in Angriff genommen ist. 
8. Es ist auf Grund der mitgeteilten Tierversuche Aussicht vorhanden, nach Auffindung von wirksamen $\mathrm{Hg}$-Verbindungen mit chemotherapeutischem Index auf dem Wege der einzeitigen intravenösen Einverleibung von Salvarsanpräparaten mit $\mathrm{Hg}$-Verbindungen eine echte Kombinationswirkung und so eine weitere Verbesserung der Salvarsantherapie zu erzielen.

\section{Sitzung am 16. Mai 1921 nachmittags 3 Uhr. Diskussion zu "Syphilis und Liquor".}

Herren A. Buschke und Ernst Sklarz-Berlin: Erfahrungen über Syphilis des Nervensystems unter Berücksichtigung des Liquorbefundes.

Das gehäufte Auftreten der Nervenlues, das wohl jetzt kaum noch bezweifelt wird, und das seinen Grund in tatsächlich erheblicherem Vorkommen hat, zum Teil natürlich auch infolge der Verfeinerung der Untersuchungsmethoden öfters als früher diagnostiziert wird, hat uns die Veranlassung gegeben, das diesbezügliche Material des Städtischen Rudolf Virchow-Krankenhauses zu Berlin zusammenfassend durchzusehen. Es umfaßt zunächst die Fälle der eigenen Abteilung, andererseits durch das freundliche Entgegenkommen der dirigierenden Ärzte der beiden inneren und der ophthalmologischen Abteilung (Geh.-Rat Kuttner, Geh.-Rat Brandenburg, Prof. Fehr) auch die Nervenlueskranken, die auf deren Stationen in der Zeit von 1907 bis 1920 einschließlich in Behandlung gewesen sind. Es war uns aus äußeren Gründen bisher noch nicht möglich, alle Fälle durchzuarbeiten, doch konnten wir immerhin bis jetzt 7239 Luetiker bzw. deren Krankengeschichten verwenden. Der Rest von weiteren ca. 1500 wird wohl in absehbarer Zeit bewältigt sein und dann in Verbindung mit den bereits verwerteten Fällen zur Veröffentlichung gelangen.

Jedenfalls ist dureh die Vielseitigkeit des Materials eine größere Gleichmäßigkeit des Überblicks gewährleistet, als wenn nur die Fälle der Dermatologischen Abteilung, der häufig die reinen Neurofälle ohne Erscheinungen entgehen, oder diejenigen der inneren Stationen, bei denen das Gegenteil der Fall ist, zur Verwendung gekommen wären. Die Hinzunahme der spezifischen Augenerkrankungen schließt den Kreis so ziemlich, da wohl nur wenig luetische Ohrenerkrankungen nicht wenigstens ambulant fachärztlich auf der betreffenden Station untersucht wurden und herangezogen werden konnten. Die Zahl der Nervenluesfälle auf den chirurgischen Abteilungen ist einerseits an sich sehr gering, andererseits werden derartige Kranke, wenn die Syphilis des Nervensystems erst einmal diagnostiziert ist, doch auf die inneren bzw. dermatologischen Stationen verlegt ${ }^{1}$ ).

Unter den 7239 Luesfällen fjnden sich nach unserer bisherigen Feststellung $316=4,2 \%$ Kranke mit Erscheinungen von seiten des Nervensystems, und zwar Lues cerebri bzw. Lues cerebrospinalis. . . . . . . . . . . $152=2,1 \%$ Tabes . . . . . . . . . . . . . . . $127=1,7 \%$ Paralyse . . . . . . . . . . . . . . . . . . 24 $24=0,3 \%$ Taboparalyse . . . . . . . . . . . . . . $13=0,1 \%$

1) Ein Teil der Fälle wurde von dem neurologischen Consiliarius unseres Krankenhauses, Herrn Priv.-Doz. Dri. Simons, untersucht, dem wir auch an dieser Stelle für seine Bemühungen und Unterweisungen bestens danken. 